\title{
Fractional Fourier transform as a signal processing tool: An overview of recent developments
}

\author{
Ervin Sejdić, Igor Djurović and LJubiša Stanković
}

\begin{abstract}
Fractional Fourier transform (FRFT) is a generalization of the Fourier transform, rediscovered many times over the past hundred years. In this paper, we provide an overview of recent contributions pertaining to the FRFT. Specifically, the paper is geared toward signal processing practitioners by emphasizing the practical digital realizations and applications of the FRFT. It discusses three major topics. First, the manuscripts relates the FRFT to other mathematical transforms. Second, it discusses various approaches for practical realizations of the FRFT. Third, we overview the practical applications of the FRFT. From these discussions, we can clearly state the FRFT is closely related to other mathematical transforms, such as time-frequency and linear canonical transforms. Nevertheless, we still feel that major contributions are expected in the field of the its digital realizations and applications, especially, since many digital realizations of the FRFT still lack properties of the continuous FRFT. Overall, the FRFT is a valuable signal processing tool. Its practical applications are expected to grow significantly in years to come, given that the FRFT offers many advantages over the traditional Fourier analysis.
\end{abstract}

\section{INTRODUCTION}

In very simple terms, the fractional Fourier transform (FRFT) is a generalization of the ordinary Fourier transform [1]. Specifically, the FRFT implements the so-called order parameter $\alpha$ which acts on the ordinary Fourier transform operator. In other words, the $\alpha$ th order fractional Fourier transform represents the $\alpha$ th power of the ordinary Fourier transform operator. When $\alpha=\pi / 2$, we obtain the Fourier transform, while for $\alpha=0$, we obtain the signal itself. Any intermediate value of $\alpha(0<\alpha<\pi / 2)$ produces a signal representation that can be considered as a rotated time-frequency representation of the signal [2],

Signal Processing, Vol. 91 No. 6, June, 2011
$[3]$.

Interestingly enough, the idea of the fractional powers of the Fourier operator has been "discovered" several times in the literature. Initially, the idea appeared in the mathematical literature between the two world wars (e.g., [4], [5]). More publications relating to this idea appeared after the second world war, however they were sporadic (e.g. [6]). The idea of fractional Fourier operator re-gains a momentum in 1980's with publications by Namias (e.g. [7]). Following Namias' contributions, a large number of papers appeared in the literature during 1990's tying the concept of the fractional Fourier operators to many other fields (e.g., time-frequency analysis as described in $[2]$ ). We have also witnessed a number of recent contributions attempting to understand the practical applications of the FRFT beyond optics.

The main goal of this publication is to provide an overview of recent developments regarding the FRFT and its applications. Although a number of publications reviewing the FRFT has also appeared in recent years (e.g., [1], [8], [9]), some of these publications are geared towards explaining the mathematical eloquence behind the FRFT. Our goal is to simplify the theory behind the FRFT and provide an overview suitable for a signal processing practitioner. In particular, we emphasize the practicality of the FRFT by devoting a significant part of this manuscript to its discrete realizations and applications. By attracting more practitioners to this eloquent mathematical concept, we hope to foster more applications since the FRFT offers many valuable properties, which otherwise were not available with traditional tools (e.g., the Fourier trans- 
form).

The paper is organized as follows: Section II introduces the concept of FRFT and relates the transform to other mathematical representations. In Section III, we discuss several various implementation approaches for discrete signals. Section IV provides an overview of some FRFT applications, while in Section V, we provide concluding remarks along with an outline of possible future directions.

\section{The Fractional Fourier transform}

The fractional Fourier transform (FRFT) is a linear operator defined as [10]-[13]:

$$
X_{\alpha}(u)=\mathcal{F}_{\alpha}(x(t))=\int_{-\infty}^{+\infty} x(t) K_{\alpha}(t, u) d t
$$

with $K_{\alpha}(t, u)$ representing the kernel function defined as:

$$
\begin{array}{cc}
K_{\alpha}(t, u) & \\
& \text { if } \alpha \text { is } \\
\sqrt{\frac{1-j \cot \alpha}{2 \pi}} e^{j\left(u^{2} / 2\right) \cot \alpha} & \text { not } \\
\times e^{j\left(t^{2} / 2\right) \cot \alpha-j u t \csc \alpha} & \text { multiple } \\
& \text { of } \pi \\
\delta(t-u) & \text { if } \alpha \text { is } \\
& \text { a multiple } \\
& \text { of } 2 \pi \\
\delta(t+u) & \text { if } \alpha+\pi \text { is } \\
& \text { a multiple } \\
& \text { of } 2 \pi
\end{array}
$$

and $\delta(t)$ representing the Dirac function. Throughout the paper we use $\mathcal{F}_{\alpha}$ to denote the operator associated with the FRFT. It should be noted that we adopted notation for the FRFT found in signal processing literature. In particular, we denote the rotation angle by $\alpha$. Mathematicians usually denote the rotation angle by $a$, where $a=2 \alpha / \pi$. It is important to point out that various $\alpha$ values provide transformations with distinctive properties. Hence, $\alpha$ can be adjusted in many applications to provide enhanced results in comparison to other existing methods. Additional computational complexity associated with such optimization efforts is often acceptable.

Given the properties of the kernel, equation (1) is equal to $x(t)$ when $\alpha$ is a multiple of $2 \pi$, and is equal to $x(-t)$ when $\alpha+\pi$ is a multiple of $2 \pi$. Some properties associated with the FRFT are summarized below [1], [14]:

1. The standard Fourier transform is a special case of the FRFT with a rotation angle $\alpha=$ $\pi / 2$.

2. $\mathcal{F}_{0}$ is the identity operator, i.e., $\mathcal{F}_{0}(x(t))=$ $x(t)$. The same can be stated for $\mathcal{F}_{2 \pi}$.

3. The FRFT is a linear operator, i.e., $\mathcal{F}_{\alpha}\left(\sum_{k} c_{k} x_{k}(t)\right)=\sum_{k} c_{k} \mathcal{F}_{\alpha}\left(x_{k}(t)\right)$.

4. The FRFT adheres to commutativity (i.e., $\mathcal{F}_{\alpha_{1}} \mathcal{F}_{\alpha_{2}}=\mathcal{F}_{\alpha_{2}} \mathcal{F}_{\alpha_{1}}$ ) and associativity (i.e., $\left.\left(\mathcal{F}_{\alpha_{3}} \mathcal{F}_{\alpha_{2}}\right) \mathcal{F}_{\alpha_{1}}=\mathcal{F}_{\alpha_{3}}\left(\mathcal{F}_{\alpha_{2}} \mathcal{F}_{\alpha_{1}}\right)\right)$.

5. Successive applications of the FRFT of various orders is equal to a single application of the FRFT whose order is equal to the sum of individual orders (e.g., $\mathcal{F}_{\alpha_{1}} \mathcal{F}_{\alpha_{2}}=\mathcal{F}_{\alpha_{1}+\alpha_{2}}$ ).

6. Inverse FRFT is obtained by applying $\mathcal{F}_{-\alpha}$ to the transformed signal (i.e., $\mathcal{F}_{\alpha} \mathcal{F}_{-\alpha}=\mathcal{F}_{0}$ ). 7. FRFT satisfies the Parseval's theorem: $\langle x(t), y(t)\rangle=\left\langle X_{\alpha}(u), Y_{\alpha}(u)\right\rangle$.

Additional properties of the FRFT and some transform pairs are listed in Tables I and II. These properties clearly indicate that the FRFT is an extension of the ordinary Fourier transform. More extensive descriptions and proofs of these properties, along with additional transform pairs, can be found in other references (e.g., [1], [10], [11], [12]). The FRFTs of a few sample signals are shown in Figure 1.

A particularly interesting case of the above listed properties is the fact that it can be shown that the FRFT of a product of two signals, $y(t)=x(t) w(t)$, can be shown to be equal to $[15]$ :

$$
\begin{aligned}
& Y_{\alpha}(u)=\frac{|\csc \alpha|}{\sqrt{2 \pi}} \exp \left(\frac{j u^{2} \cot \alpha}{2}\right) \int_{-\infty}^{+\infty} X_{\alpha}(v) \\
& \times W((u-v) \csc \alpha) \exp \left(-\frac{j v^{2} \cot \alpha}{2}\right) d v \quad(3)
\end{aligned}
$$

where $W(v)$ is the Fourier transform of $w(t)$. In other words, the resultant FRFT is equal to convolution of the FRFT of $x(t)$ with the Fourier transform of $w(t)$ multiplied by a chirp function. A similar expression can be derived for convolution of two signals $(y(t)=x(t) * w(t))$. In particular, convolution of two signals in time domain equals to 
TABLE I

Properties of The FRFT

\begin{tabular}{l|l}
\hline \hline Signal & FRFT \\
\hline$x(t-\tau)$ & $\exp \left(j\left(\tau^{2} / 2\right) \sin \alpha \cos \alpha-j u \tau \sin \alpha\right) X_{\alpha}(u-\tau \cos \alpha)$ \\
\hline$x(t) \exp (j v t)$ & $\exp \left(-j v^{2}(\sin \alpha \cos \alpha) / 2+j v \cos \alpha\right) X_{\alpha}(u-v \sin \alpha)$ \\
\hline$x(t) t$ & $u \cos \alpha X_{\alpha}(u)+j \sin \alpha X_{\alpha}^{\prime}(u)$ \\
\hline$x(t) / t$ & $-j \sec \alpha \exp \left(j\left(u^{2} / 2\right) \cot \alpha\right) \int_{-\infty}^{u} x(z) \exp \left(-j\left(z^{2} / 2\right) \cot \alpha\right) d z$ \\
\hline$x(c t)$ & $\sqrt{\frac{1-j \cot \alpha}{c^{2}-j \cot \alpha}} \exp \left(j\left(u^{2} / 2\right) \cot \alpha\left(1-\left(\cos ^{2} \beta / \cos ^{2} \alpha\right)\right)\right) X_{\beta}\left(\frac{u \sin \beta}{c \sin \alpha}\right) \quad$ where \\
& $\cot \beta=\frac{\cot \alpha}{c^{2}}$ \\
\hline$x^{\prime}(t)$ & $X_{\alpha}^{\prime}(u) \cos \alpha+j u \sin \alpha X_{\alpha}(u)$ \\
\hline $\int_{b}^{t} x\left(t^{\prime}\right) d t^{\prime}$ & $\begin{array}{l}\sec \alpha \exp \left(-j\left(u^{2} / 2\right) \tan \alpha\right) \int_{b}^{u} X_{\alpha}(z) \exp \left(j\left(z^{2} / 2\right) \tan \alpha\right) d z \text { if } \alpha-\pi / 2 \text { is } \\
\text { not a multiple of } \pi\end{array}$ \\
\hline
\end{tabular}

TABLE II

FRFT OF SOME BASIC FUNCTIONS.

\begin{tabular}{l|c}
\hline \hline Signal & FRFT \\
\hline 1 & $\sqrt{1+j \tan \alpha} \exp \left(-j\left(u^{2} / 2\right) \tan \alpha\right)$ \\
\hline$\delta(t-\tau)$ & $\sqrt{\frac{1-j \cot \alpha}{2 \pi}} \exp \left(j\left(\left(\tau^{2}+u^{2}\right) / 2\right) \cot \alpha-j u \tau \operatorname{cosec} \alpha\right)$ \\
\hline $\exp \left(-t^{2} / 2\right)$ & $\exp \left(-u^{2} / 2\right)$ \\
\hline $\exp (j \eta t)$ & $\sqrt{1+j \tan \alpha} \exp \left(j\left(\left(\eta^{2}+u^{2}\right) / 2\right) \tan \alpha-j u \eta \sec \alpha\right)$ \\
\hline $\exp \left(j c\left(-t^{2} / 2\right)\right)$ & $\sqrt{\frac{1-j \cot \alpha}{c-j \cot \alpha}} \exp \left(j\left(u^{2} / 2\right)\left(c^{2}-1\right) \cot \alpha /\left(c^{2}+\cot ^{2} \alpha\right)\right)$ \\
& $\exp \left(-c\left(u^{2} / 2\right) \operatorname{cosec}^{2} \alpha /\left(c^{2}+\cot ^{2} \alpha\right)\right)$ \\
\hline
\end{tabular}

$[15]:$

$$
\begin{gathered}
Y_{\alpha}(u)=|\sec \alpha| \exp \left(\frac{j u^{2} \tan \alpha}{2}\right) \int_{-\infty}^{+\infty} X_{\alpha}(v) \\
\times w((u-v) \sec \alpha) \exp \left(-\frac{j v^{2} \tan \alpha}{2}\right) d v \quad(4)
\end{gathered}
$$

which shows that the FRFT of a convolution can therefore be obtained by taking the FRFT of one of the signals, multiplying by a chirp, convolving with a scaled version of the other signal, and multiplying again by a chirp and by a scale factor. These two rules are of particular interests when developing a time-frequency representation based on the FRFT.

Our next task is to understand the relationship between the FRFT and other mathematical representations. First, we begin by establishing the relationships with the ordinary Fourier transform. Then, we establish relationships with various time-frequency representations and linear canonical analysis.

\section{A. FRFT as fractional powers of Fourier transform}

In 1980, Namias provided an elegant generalization of the Fourier transform to the FRFT [7], by deriving the FRFT from the eigenfunctions of the Fourier transform. In fact, Namias used the fact that the HermiteGaussian functions $\left(\phi_{k}(t)\right)$ are eigenfunctions of the Fourier transform and showed that the $\alpha$ th order FRFT shares the eigenfunctions of the Fourier transform. More specifically, the eigenvalues of the FRFT are the $\alpha$ th root of the eigenvalues of the Fourier transform [7]:

$$
\mathcal{F}_{\alpha}\left(\phi_{k}(t)\right)=\exp (-j \alpha k) \phi_{k}(t)
$$

Therefore, if we expand a signal in terms of these eigenfunctions, we obtained

$$
x(t)=\sum_{k=0}^{\infty} c_{k} \phi_{k}(t)
$$



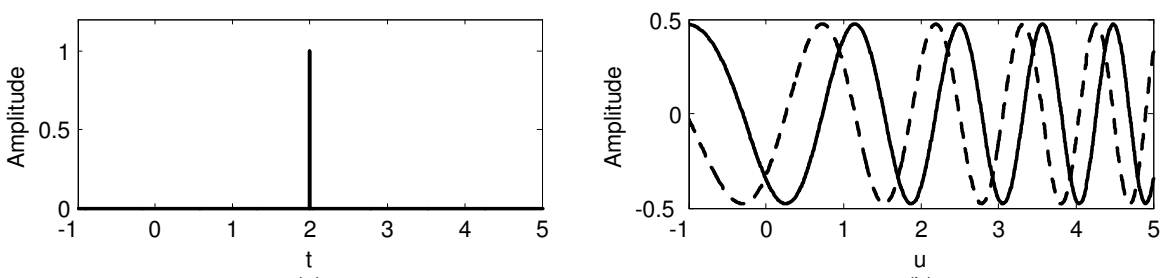

(a)
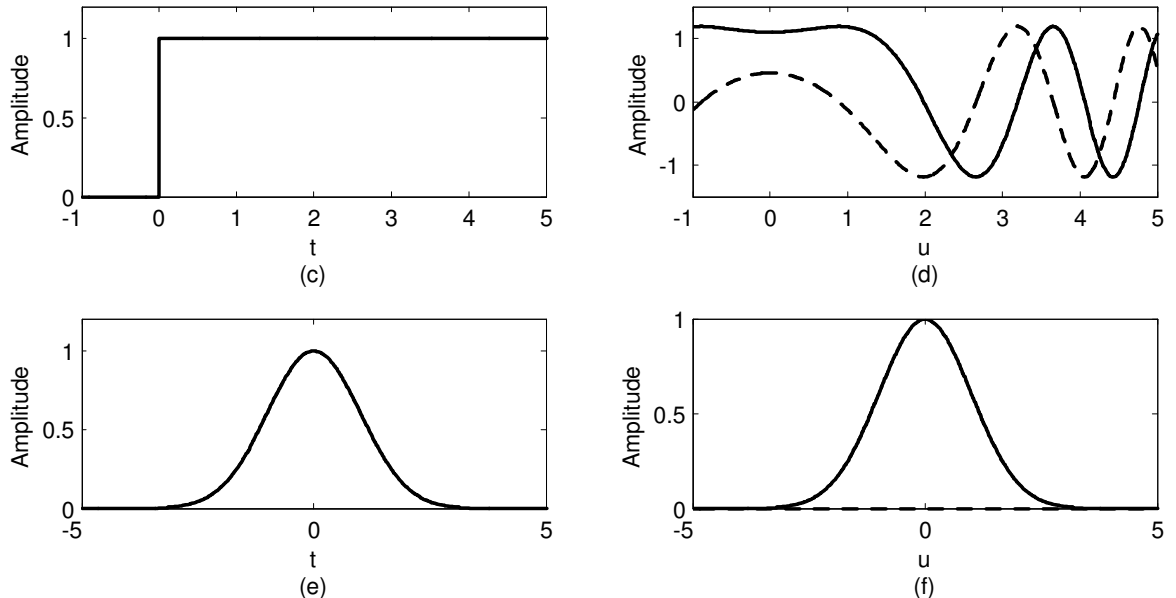

Fig. 1. Sample signals and their theoretical FRFTs $(\alpha=\pi / 4)$ : (a) time domain representation of the Dirac functio; (b) FRFT of the Dirac function; (c) time domain representation of the unit function; (d) FRFT of the unit function; (e) time domain representation of the exponential function; (f) FRFT of the exponential function. Solid lines represent real parts, while dashed lines represent imaginary parts.

where

$$
c_{k}=\int_{-\infty}^{+\infty} \phi_{k}(t) x(t) d t
$$

By applying the fractional operator $\left(\mathcal{F}_{\alpha}(\cdot)\right)$ to both sides of (6), we get

$$
\begin{gathered}
\mathcal{F}_{\alpha}(x(t)) \\
=\sum_{k=0}^{\infty} c_{k} \mathcal{F}_{\alpha}\left(\phi_{k}(t)\right) \\
=\sum_{k=0}^{\infty} c_{k} \exp (-j \alpha k) \phi_{k}(u) \\
=\int \sum_{k=0}^{\infty} \exp (-j \alpha k) \phi_{k}(u) \phi_{k}(t) x(t) d t
\end{gathered}
$$

From the above equation, it is clear that the kernel function can be defined as:

$$
K_{\alpha}(t, u)=\sum_{k=0}^{\infty} \exp (-j \alpha k) \phi_{k}(u) \phi_{k}(t)
$$

The above equation can be considered as the spectral decomposition of the kernel function of FRFT [1]. Using the Mehler's formula, Namias in fact shows that we obtain the FRFT from (8) [7].

\section{B. Relations to time-frequency representa- tions}

To understand the relationship between the FRFT and various time-frequency analysis, we consider the relationship between the ambiguity function and the FRFT. The ambiguity function (AF) of a signal, $x(t)$, is

$$
\begin{aligned}
A F_{x}(t, f)= & \int_{-\infty}^{+\infty} x\left(\tau+\frac{t}{2}\right) x^{*}\left(\tau-\frac{t}{2}\right) \\
& \times \exp (-j 2 \pi \tau f) d \tau .
\end{aligned}
$$

By introducing rotation of the AF by using the following transformation:

$$
\begin{aligned}
& t=R \cos \alpha \\
& f=R \sin \alpha
\end{aligned}
$$


with $R \in(-\infty, \infty)$ and $\alpha \in[0, \pi)$, and redefining the $\mathrm{AF}$ as $F R A F_{x}(R, \alpha)=$ $A F_{x}(R \cos \alpha, R \sin \alpha)$, it becomes obvious that the FRFT corresponds to a rotation of the AF [16]. In particular, it is easy to prove that the fractional power spectra is the Fourier transform of the AF:

$$
\begin{gathered}
F R A F_{x}\left(R, \alpha-\frac{\pi}{2}\right) \\
=\int_{-\infty}^{+\infty}\left|X_{\alpha}(u)\right| \exp (j 2 \pi R u) d u .
\end{gathered}
$$

Using this relationship, the connection between the local fractional FT moments and the angle derivative of the fractional power spectra has been established in [17]. Furthermore, it can be easily generalized that the FRFT corresponds to rotation of a class of time-frequency representations (TFRs) as along as $\Psi(t, f)=\mathcal{F}_{\theta \rightarrow t, \tau \rightarrow f}^{-1}\{\phi(\theta, \tau)\}$ is rotational symmetric [18]-[21], where $\phi(\theta, \tau)$ is a two-dimensional kernel function. For example, the FRFT corresponds to the rotation of the Wigner distribution (WD) in the timefrequency plane [20], [22]-[25]:

$$
\begin{gathered}
W D_{F_{\alpha}(x(t))}(t, f) \\
=W D_{x}(t \cos \alpha-f \sin \alpha, u \sin \alpha+f \cos \alpha) .
\end{gathered}
$$

The relationship between FRFT and RadonWigner distribution (RWD) (e.g., [26]) is studied in [23], [27], and it was shown that the Radon-Wigner distribution is the squared modulus of the fractional Fourier transform:

$$
R W[x(t)]=\left|\mathcal{F}_{\alpha}[x(t)]\right|^{2} .
$$

Additionally, we should point out that the FRFT could be potentially useful for obtaining time-frequency distributions without cross-terms. For example, WD and AF exhibit different behaviors when considering signals with multiple components. Namely, the WD has signal components (i.e., auto-terms) concentrated around their instantaneous frequencies and group delays, while interferences (cross-terms) are located between these components. On the other hand, the AF has the auto-terms concentrated around the origin while the interferences are dislocated from the origin. The connection between these two time-frequency representations is the $2 \mathrm{D}$ Fourier transform:

$$
W D_{x}(t, \omega)=F T_{\tau \rightarrow \omega, \theta \rightarrow t}\left\{A F_{x}(\theta, \tau)\right\} .
$$

However, interesting results are obtained when we use the 2D FRFT instead of the 2D Fourier transfrom in (16). Specifically, it is possible to separate all auto- and cross-terms in the timefrequency plane for some classes of signals. To illustrate this behaviour, let us considered a signal with 4 components concentrated in the $\mathrm{TF}$ plane around $(t, f)=( \pm 0.5, \pm 6)$, and the signal is defined as:

$$
\begin{gathered}
x(t)=\left[\exp \left(-60(t-0.5)^{2}\right)\right. \\
\left.+\exp \left(-60(t+0.5)^{2}\right)\right][\exp (j 12 \pi t)+\exp (-j 12 \pi t)]
\end{gathered}
$$

with $t \in[-1,1]$. The AF of this signal is depicted in Figure 2(a). All four auto-terms are located in the middle of the graph (i.e., the origin of the ambiguity plane). Each of four horizontal and vertical objects with respect to the origin represents two cross-terms, while the corner objects represent single cross-terms. In the case of the WD (Figure 2(b)), the corner objects are auto-terms, while the central object represents the four cross-terms. The remaining objects contain two cross terms each. However, all 16 components (4 auto terms and 12 cross-terms) are clearly separated as shown in Figure 2(c) when we used the FRFT operator with $\alpha=0.5$ in (16).

\section{B.1 Local polynomial Fourier transform}

It is also of our interest to establish the relationship between the FRFT and the local polynomial Fourier transform (LPFT) ([28], [29]). First, we rewrite the FRFT as:

$$
\begin{aligned}
& \mathcal{F}_{\alpha}(x(t))=\sqrt{\frac{1-j \cot \alpha}{2 \pi}} \\
& \times e^{j\left(u^{2} / 2\right) \cot \alpha} \int_{-\infty}^{+\infty} x_{w}(\tau) \\
& \times e^{j\left(\tau^{2} / 2\right) \cot \alpha-j u \tau \csc \alpha} d \tau
\end{aligned}
$$

where $x_{w}(\tau)=x(t+\tau) w(\tau)$. If the LPFT is given by [28], [29]:

$$
\operatorname{LPFT}_{x}(t, \bar{f})=\int_{-\infty}^{+\infty} x(t+\tau)
$$



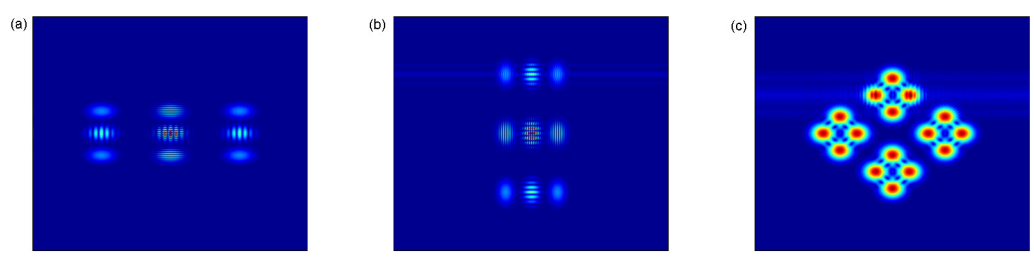

Fig. 2. Time frequency representation of the four-component signal: (a) the AF; (b) the WD; (c) the FRFT of the AF.

$$
\begin{gathered}
w(\tau) \exp \left(-j 2 \pi f_{1} \tau-j 2 \pi f_{2} \tau^{2} / 2\right. \\
\left.-\ldots-j 2 \pi f_{M} \tau^{M} / M\right) d \tau
\end{gathered}
$$

where $\bar{f}=\left(f_{1}, f_{2}, \ldots, f_{M}\right)$, we set $M=2$, $2 \pi f_{1}=u \csc \alpha$, and $2 \pi f_{2}=\cot \alpha$, then (18) can be expressed in terms of the LPFT as:

$$
\begin{array}{r}
\mathcal{F}_{\alpha}(x(t))=\sqrt{\frac{1-j \cot \alpha}{2 \pi}} \\
\times e^{j\left(u^{2} / 2\right) \cot \alpha}{ } P F T_{x}\left(t, f_{1}, f_{2}\right) .
\end{array}
$$

From these equations it can be clearly seen that the LPFT provides a broad generalization of the FRFT. Similar observations have been made in [30]. Additionally, we can relate the FRFT to the local polynomial periodogram (LPP), which is defined as the squared magnitude of the LPFT:

$$
\operatorname{LPP}_{x}(t, \bar{f})=\left|\operatorname{LPFT}_{x}(t, \bar{f})\right|^{2} .
$$

To understand the relationship between the FRFT and the LPP, let us consider the LPP in more details. The LPP is a distribution belonging to the Cohen class of distributions [28], [29]. Its kernel is defined as the $\mathrm{AF}$ of $w_{g}(t)=w(t) \exp \left(-j 2 \pi f_{2} t^{2} / 2-\ldots-\right.$ $j 2 \pi f_{M} t^{M} / M$ !), which is a generalization of the kernel used for the spectrogram (i.e., the squared magnitude of the short-time Fourier transform), where the kernel function is defined as the $\mathrm{AF}$ of $w(t)$. This generalization stems from the fact that the short-time Fourier transform is equal to the LPFT for $M=1$. Hence, to relate the LPP to (20), let us consider the second order case (i.e., $M=2$ ):

$$
A F_{w_{g}}(t, f)=\int_{-\infty}^{+\infty} w\left(\tau+\frac{t}{2}\right) w^{*}\left(\tau-\frac{t}{2}\right)
$$

$$
\begin{gathered}
\times \exp \left(-j 2 \pi f_{2}(\tau+t / 2)^{2}\right. \\
\left.\left.+j 2 \pi f_{2}(\tau-t / 2)^{2}-j 2 \pi f \tau\right)\right) d \tau \\
=\int_{-\infty}^{+\infty} w\left(\tau+\frac{t}{2}\right) w^{*}\left(\tau-\frac{t}{2}\right) \\
\times \exp \left(-j 4 \pi f_{2} \tau t-j 2 \pi f \tau\right) d \tau \\
=A F_{w}\left(t, f+2 \pi f_{2} t\right),
\end{gathered}
$$

where $A F_{w}(t, f)$ is the ambiguity function of the used window. Hence, the LPP rotates $A F_{w}(t, f)$ in the ambiguity plane and can adjust the resulting time-frequency representation according to the signal of interest. Similarly can be stated for any localized form of the FRFT with a proper selection of the $\alpha$ (angle) parameter.

Equations (13)-(22) demonstrate that various coordinate transformations have been implemented to relate the FRFT to different transformations. This implies that any coordinate transformation can be used, which is demonstrated in the next section, where we relate the FRFT with the linear canonical transform.

\section{B.2 Other time-frequency representations and properties}

A formal relationship between the wavelet transform and the FRFT has also been established [31], [32]. Specifically, the FRFT kernels corresponding to different values of $\alpha$ are closely related to the wavelet transform, which can be obtained from the quadratic phase function in (2) by scaling the coordinates and the amplitude [31], [32]. Furthermore, fractional-Fourier-domain realizations of several other time-frequency representations were also introduced. These include realizations of the short-time Fourier transform [33], the 
weighted Wigner distribution (i.e., S-method) [21], [34], the Gabor expansion [35]-[38], and the tomography time-frequency transform defined as the inverse Radon transform of the FRFT [39].

Some of the properties associated with the time-frequency analysis have been extended to the FRFT. For example, marginals associated with time-frequency representations based on the fractional Fourier transform were examined [40] in analogy to marginals associated with TFRs based on the standard Fourier transform. However, it is not very clear what is a precise meaning of those generalized marginals in comparison to the traditional time and frequency marginals, which have a specific meaning relating those quantities to the signal under analysis. Several publications also investigated the uncertainty principle in the fractional domain [41]-[44].

\section{FRFT and linear canonical transform}

The linear canonical transform (LCT) is a multiparameter integral transform and it represents a generalization of many mathematical transformations (e.g., the Fourier transform, FRFT, Fresnel transform) [1], [45]. It should be mentioned that the LCT is also called the affine Fourier transform, the generalized Fresnel transform, the Collins formula, the ABCD transform, or the almost Fourier and almost Fresnel transformation [1], [45]. The transformation is useful in many practical applications such as optics, radar system analysis, filter design, phase retrieval, and pattern recognition [45].

The LCT is defined by [1], [45], [46]:

$$
\begin{gathered}
L C T_{\Lambda}(x(t)) \\
=\left\{\begin{array}{c}
\sqrt{\frac{1}{j 2 \pi b}} \exp \left(j d u^{2} /(2 b)\right) \\
\times \int_{-\infty}^{+\infty} \exp (-j u t / b) \\
\times \exp \left(j a t^{2} /(2 b)\right) x(t) d t \\
\sqrt{d} \exp \left(j c d u^{2} / 2\right) x(d \cdot u) \quad b=0
\end{array}\right.
\end{gathered}
$$

where $a d-b c=1, \Lambda=\{a, b, c, d\}$. Using the above equation, it is straightforward to show that the FRFT is the special case of LCT where $\{a, b, c, d\}=\{\cos \alpha, \sin \alpha,-\sin \alpha, \cos \alpha\}$ with some phase correction [1], [45], [46]. In other words,

$$
\begin{gathered}
\mathcal{F}_{\alpha}(x(t))=\sqrt{\exp (j \alpha)} \\
\times L C T_{\{\cos \alpha, \sin \alpha,-\sin \alpha, \cos \alpha\}}(x(t))
\end{gathered}
$$

Additionally, other existing forms of the LCT can be related to FRFT. For example, the simple coordinate transform proposed in [16] when considered with parameters $\{1, \alpha, 0,1\}$ represents the form of the second order polynomial Fourier transform with $2 \pi f_{2}=\alpha$. Furthermore, de Bruijn proposed a form of the LCT in 1973 [6]. The parameters of this distribution are $\{\cosh \alpha, \sinh \alpha, \sinh \alpha, \cosh \alpha\}$ and its kernel function is defined as:

$$
\begin{gathered}
K_{\alpha}(t, u)=\frac{1}{\sqrt{\sinh \alpha}} \\
\times e^{-j \pi\left(u^{2} / 2\right) \operatorname{coth} \alpha} e^{-j \pi\left(t^{2} / 2\right) \operatorname{coth} \alpha+j \pi u t / \sinh \alpha} .
\end{gathered}
$$

which is quite similar to the FRFT. This transform shares numerous properties of FRFT and it will be interesting to investigate a range of possible application of this coordinate transform given recent advances in signal processing.

C.1 Coordinate transformation of other higherorder time-frequency representations

The FRFT and linear canonical transform framework can be generalized for some higher order time-frequency representations. Here, we discuss the case of the L-Wigner distribution (L-WD) introduced in [47] for achieving high concentration of the time-frequency representations by eliminating the so-called inner interferences. The L-WD can be defined as:

$$
\begin{gathered}
L W D(t, f)=\int_{-\infty}^{+\infty} x^{L}(t+\tau / 2) \\
\times x^{* L}(t-\tau / 2) \exp (-j 2 \pi f t \tau) d \tau .
\end{gathered}
$$

Signal $x_{\Lambda}(t)$, producing the coordinate transform of the L-WD $L W D(a t+b f, c t+d f)$, can be defined for $b \neq 0$ as:

$$
\begin{gathered}
x_{\Lambda}(t)=(1 / 2 \pi|b|)^{1 / 2 L} \\
\times\left(\int_{-\infty}^{+\infty} x^{L}(t) e^{j \frac{L}{2}\left(\frac{1-a}{b}\right) u^{2}-j \frac{L(t-u)^{2}}{2 b}} d u\right)
\end{gathered}
$$




$$
\times e^{j \frac{1-d}{b} t^{2} / 2} .
$$

For rotation $a=d=\cos \alpha$ and $c=-b=\sin \alpha$, the generalized $(\mathrm{L})$ form of the FRFT can be written as:

$$
\begin{gathered}
X_{L, \alpha}(u)=\left(\frac{L(1-j \cot \alpha)}{2 \pi}\right)^{1 / 2 L} \\
\times\left(\int_{-\infty}^{+\infty} x^{L}(t) e^{j \frac{L}{2} \cot \alpha\left(u^{2}+t^{2}\right)-j L u t \csc \alpha} d t\right)^{1 / L} .
\end{gathered}
$$

The L-FRFT form can be evaluated using the FRFT as:

$$
\begin{gathered}
X_{L, \alpha}(u)=L^{1 / 2 L}\left(\frac{1+j \cot \alpha}{2 \pi}\right)^{\frac{L-1}{2 L}} \\
\times\left(\int_{-\infty}^{+\infty} \int_{-\infty}^{+\infty} \cdots \int_{-\infty}^{+\infty}\left(\prod_{i=1}^{L} X_{\alpha}\left(u_{i}\right) d u_{i}\right)\right. \\
\left.\times e^{-j \frac{\cot \alpha}{2}\left(\sum_{i=1}^{L} u_{i}^{2}-u^{2}\right)} \delta\left(L u-\sum_{i=1}^{L} u_{i}^{2}\right)\right) .
\end{gathered}
$$

For $L=2$, this relationship can be written as:

$$
\begin{gathered}
X_{2, \alpha}(u)=\left(\frac{1+j \cot \alpha}{\pi}\right)^{1 / 4} \\
\times\left(\int_{-\infty}^{+\infty} X_{\alpha}(u+\tau) X_{\alpha}^{*}(u-\tau) e^{-j \cot \alpha \tau^{2}} d \tau\right)^{1 / 2}
\end{gathered}
$$

In general the L-FRFT of the $k+m$-th order can always be expressed using the L-FRFT of $k$-th and $m$-th orders:

$$
\begin{gathered}
X_{k+m, \alpha}(u)=\left(\frac{(k+m)(1+j \cot \alpha)}{2 \pi k m}\right)^{1 / 2(k+m)} \\
\times\left(\int_{-\infty}^{+\infty} X_{k, \alpha}^{k}(u+\tau / k) X_{m, \alpha}^{m}(u-\tau / m)\right. \\
\left.\times e^{-j\left(\frac{k+m}{k m}\right) / 2} d \tau\right)^{1 /(k+m)} .
\end{gathered}
$$

Similar coordinate transform can be determined for all time-frequency representations depending on powers of the signal localautocorrelation $x(t+\tau / 2) x^{*}(t-\tau / 2)$. However, as far as we know there are no reported results for other higher-order time-frequency representations such as the polynomial Wigner distribution (e.g., [48]) with the usage of several auto-correlations.
C.2 Multiparameter coordinate transformations

Further generalizations can be achieved by introducing additional parameters. Mathematically, such generalization would be given as

$$
\begin{gathered}
{\left[\begin{array}{l}
t^{\prime} \\
f^{\prime}
\end{array}\right]=\left[\begin{array}{ll}
a & b \\
c & d
\end{array}\right]\left[\begin{array}{l}
t \\
f
\end{array}\right]} \\
+\left[\begin{array}{ll}
\beta & \gamma \\
\theta & \phi
\end{array}\right]\left[\begin{array}{l}
t \\
f
\end{array}\right]\left[\begin{array}{ll}
t & f
\end{array}\right]\left[\begin{array}{l}
1 \\
1
\end{array}\right]
\end{gathered}
$$

where $t^{\prime}$ and $f^{\prime}$ represent the transformed coordinates. These transformations correspond to more complex distortions in the timefrequency plane in comparison to the FRFT and LPFT. The generalization, as shown in (32), could be used to describe many existing transforms as well. For example, when $d \neq 0$, $\phi \neq 0$ and all other parameters are equal to zero, we obtain the LPFT for $M=3$, as shown in (19).

\section{Practical Realizations of FRFT}

In order to practically realize the FRFT based operators, filters, correlators, and other optical systems, we are required to numerically calculate the FRFT [49]. As depicted in the previous section, the FRFT is a subclass of integral transformations characterized by quadratic complex exponential kernels. These complex exponential kernels often introduce very fast oscillations as shown in Figure 3. Hence, it is not possible to evaluate these transformations by direct numerical integration since these fast oscillations require excessively large sampling rates. A possible approach is to decompose these integral transformations into sub-operations. However, we still require significantly higher sampling rates than the Nyquist rate, depending on the order and particular decomposition employed. This results in greater time of computation, larger numerical inaccuracies, and the need for more memory. Therefore, the goal of this section is to provide an overview of various practical implementations proposed over the years. Given that we are concerned with practical implementations using discrete signals, we only consider approaches for the implementation of the so-called discrete fractional Fourier transform 
(DFRFT). In other words, for a discrete signal, $x(n)$, of length $N$ we can define the DFRFT as follows:

$$
\mathbf{X}_{\alpha}(n)=\mathbf{F}_{\alpha} \mathbf{x}(n)
$$

where $\mathbf{F}_{\alpha}$ is defined as the discrete fractional Fourier transform matrix, $\mathbf{x}(n)$ is a vector representing the signal, and $\mathbf{X}_{\alpha}(n)$ is a vector representing the DFRFT of the signal. Therefore, the optical based implantations (e.g., [49]) are beyond the scope of this paper. A summary of various approaches is shown in Table III.

\section{A. DFRFT through sampling of FRFT}

A straightforward approach for obtaining the DFRFT is to sample the FRFT, since the sampling theorems for the FRFT of bandlimited and time-limited signals follow from those of the Shannon sampling theorem [14], [50][55]. However, the resultant discrete transform may lose many important properties (i.e., unitarity and reversibility). In addition, the DFRFT obtained by direct sampling of the FRFT lacks closed-form properties and is not additive, meaning that its applications are very limited [56]. In order to maintain some of FRFT properties, a type of DFRFT, derived as a special case of the continuous FRFT, was proposed in [57]. Specifically, it was assumed that the input function is a periodic, equally spaced impulse train. Since this type of DFRFT is a special case of continuous FRFT, many properties of the FRFT also exist and have the fast algorithm. However, this type of DFRFT cannot be defined for all values of $\alpha$ due to various imposed constraints. Around the same time, Ozaktas et. al proposed two innovative approaches for obtaining the DFRFT through sampling of the FRFT [58]. Both of the methods are based on the idea that we can manipulate the expression for the FRFT, such that the form can be appropriately sampled. In particular, the first method begins simply by multiplying the signal, $x(t)$, with a chirp function:

$$
g(t)=\exp \left[-j \pi t^{2} \tan (\alpha / 2)\right] x(t)
$$

followed by a chirp convolution

$$
\begin{aligned}
h(t)= & A_{\alpha} \int_{-\infty}^{+\infty} \exp [j \pi \csc (\alpha) \\
& \times(t-\tau)] g(\tau) d \tau .
\end{aligned}
$$

and the last chirp multiplication

$$
\mathbf{F}_{\alpha}(x(t))=\exp \left[-j \pi t^{2} \tan (\alpha / 2)\right] h(t)
$$

The convolution operation in the above equation can be achieved by sampling $g(t)$, and then performing the convolution using the fast Fourier transform (FFT). Hence, using the FFT for convolution, provides us with samples of $\mathbf{F}_{\alpha}(x(t))$. However, one has to keep in mind that the bandwidth and time-bandwidth product of $g(t)$ can be as large as twice that of $x(t)$. Thus, we need to sample $g(t)$ at the rate twice the original sampling rate used for $x(t)$, which means that the samples of $x(t)$ need to be interpolated. Specifically, assuming that $\mathbf{x}$ and $\mathbf{X}_{\alpha}$ denote column vectors with $N$ elements containing the samples of $x(t)$ and its DFRFT, respectively, then, in a vector notation, the above procedure can be given as

$$
\mathbf{X}_{\alpha}=\mathbf{F}_{\alpha} \mathbf{x}=\mathbf{D} \boldsymbol{\Lambda} \mathbf{H} \boldsymbol{\Lambda} \mathbf{J} \mathbf{x}
$$

where $\mathbf{D}$ and $\mathbf{J}$ are matrices representing the decimation and interpolation operation, $\boldsymbol{\Lambda}$ is a diagonal matrix that corresponds to chirp multiplication, and $\mathbf{H}$ corresponds to the convolution operation. Equation (37) provides the samples of the $\alpha$ th transform in terms of the samples of the original signal. This is a desirable property for a definition of the DFRFT matrix [58]. Very similar results were obtained in [52] as well. Sample computed FRFTs for impulse and step functions are shown in Figure 4. The second approach, proposed in [58], implements a very similar principle. However, the authors rewrote the FRFT into the form:

$$
\begin{aligned}
& X_{\alpha}=A_{\alpha} \exp \left(j \pi \cot (\alpha) u^{2}\right) \\
& \times \int_{-\infty}^{+\infty} \exp (-j 2 \pi \csc (\alpha) u t) \\
& \times[\exp (j \pi \cot (\alpha) t) x(t)] d t
\end{aligned}
$$


TABLE III

A SUMmary of PROPERTIES FOR MAIN DIRECTIONS FOR OBTAINING THE DFRFT.

\begin{tabular}{l|l|l}
\hline \hline Approach & Advantages & Disadvantages \\
\hline Sampling of FRFT & $\begin{array}{l}\text { Mostly straightforward ap- } \\
\text { plication of the Shannon } \\
\text { sampling theorem }\end{array}$ & $\begin{array}{l}\text { May loose many important } \\
\text { properties. }\end{array}$ \\
\hline Linear combination & $\begin{array}{l}\text { Simple implementation of } \\
\text { Fourier operators. }\end{array}$ & $\begin{array}{l}\text { The transform results do } \\
\text { not match the result of the } \\
\text { continuous FRFT. }\end{array}$ \\
\hline Eigenvalue decomposition & $\begin{array}{l}\text { Maintains some important } \\
\text { properties of the FRFT. }\end{array}$ & $\begin{array}{l}\text { Could lack the fast com- } \\
\text { putation algorithm. Can- } \\
\text { not be written in a closed } \\
\text { form. }\end{array}$ \\
\hline
\end{tabular}
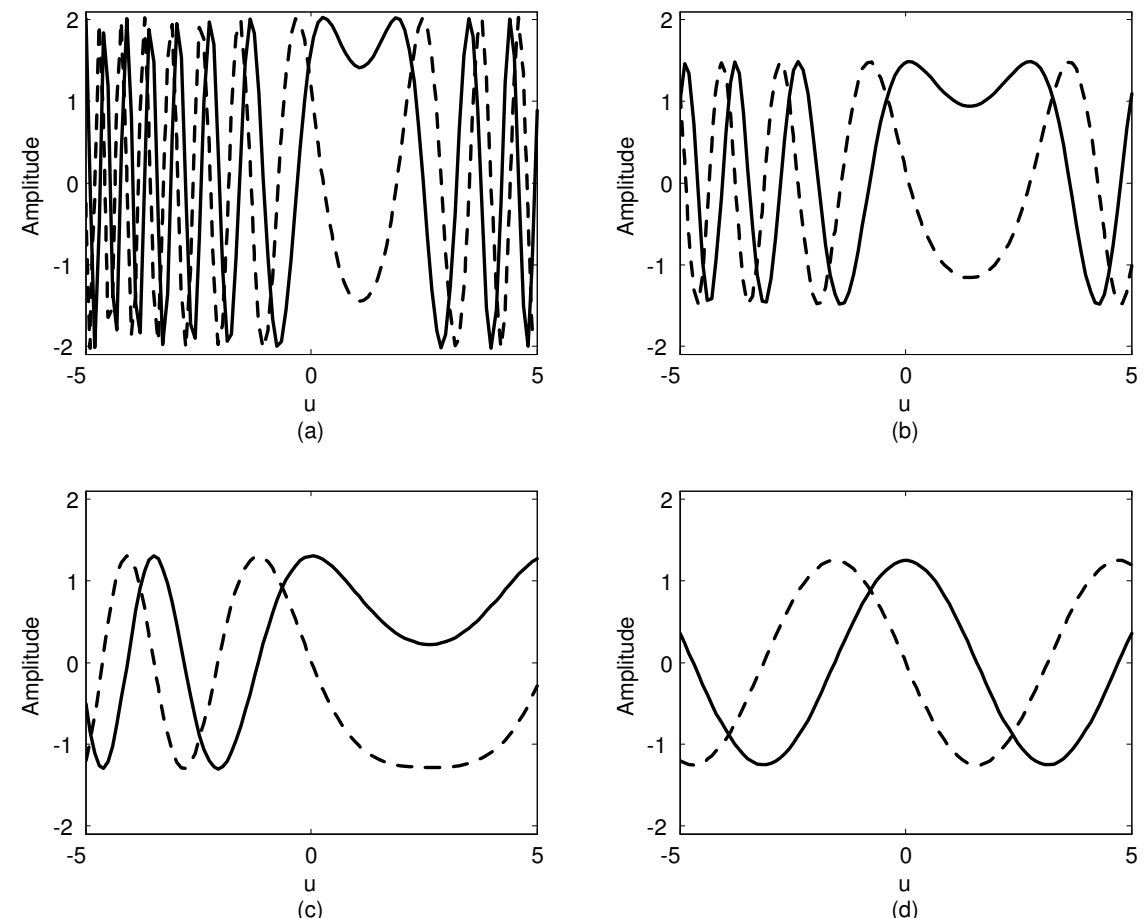

Fig. 3. The FRFT kernel function for various $\alpha$ values with the solid line representing the real part and the dashed line representing the imaginary part $(t=1)$ : (a) $\alpha=\pi / 8$; (b) $\alpha=\pi / 4$; (c) $\alpha=3 \pi / 8$; and (d) $\alpha=\pi / 2$. 

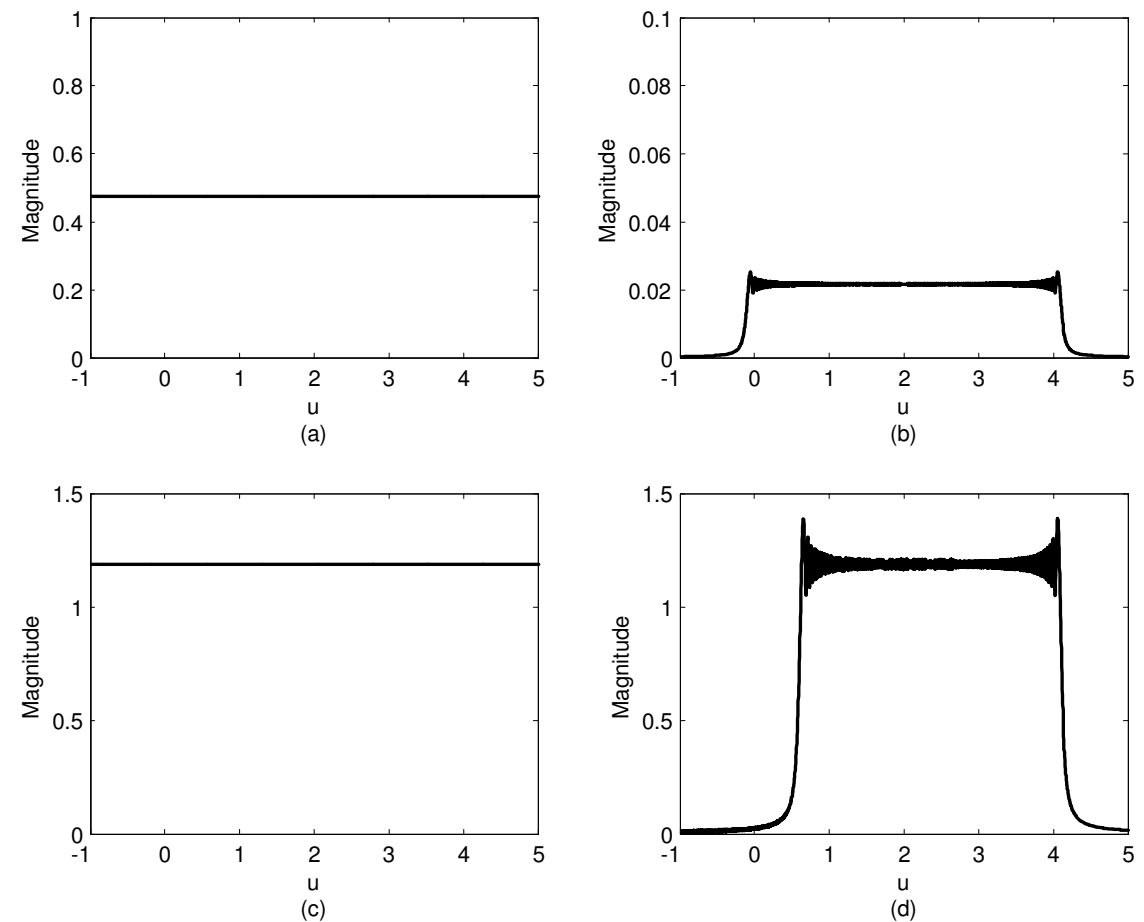

Fig. 4. Theoretical and computed FRFTs of impulse and step functions $(\alpha=\pi / 4)$ : (a) the magnitude of theoretical FRFT of the impulse function; (b) the magnitude of computed FRFT of the impulse function; (c) the magnitude of theoretical FRFT of the unit function; and (d) the magnitude of computed FRFT of the unit function.

Then, the modulated function $[\exp (j \pi \cot (\alpha) t)$ $x(t)]$ is represented by Shannon's interpolation formula:

$$
\begin{gathered}
{[\exp (j \pi \cot (\alpha) t) x(t)]} \\
=\sum_{n=-N}^{N}\left[\exp \left(j \pi \cot (\alpha) \frac{n}{2 \Delta t}\right)\right. \\
\left.\times x\left(\frac{n}{2 \Delta t}\right) \operatorname{sinc}\left(2 \Delta t\left(t-\frac{n}{2 \Delta t}\right)\right)\right]
\end{gathered}
$$

allowing us to obtain the samples of the fractional transform in terms of the samples of the original signal as

$$
\begin{gathered}
X_{\alpha}(m)=\frac{A_{\alpha}}{2 \Delta t} \sum_{n=-N}^{N} \exp (j \pi(\cot (\alpha) \\
\times\left(\frac{m}{2 \Delta t}\right)^{2}-2 \csc (\alpha) \frac{m n}{(2 \Delta t)^{2}}+ \\
\left.\left.\cot (\alpha)\left(\frac{n}{2 \Delta t}\right)^{2}\right)\right) x\left(\frac{n}{2 \Delta t}\right)
\end{gathered}
$$

which is a finite summation and $\Delta t$ represents the sampling interval. In a vector form, the overall procedure can be represented as

$$
\mathbf{X}_{\alpha}=\mathbf{F}_{\alpha} \mathbf{x}=\mathbf{D K J} \mathbf{x}
$$

where

$$
\begin{gathered}
\mathbf{K}(m, n)=\frac{A_{\alpha}}{2 \Delta t} \exp \left(j \pi \left(\cot (\alpha)\left(\frac{m}{2 \Delta t}\right)^{2}\right.\right. \\
\left.\left.-2 \csc (\alpha) \frac{m n}{(2 \Delta t)^{2}}+\cot (\alpha)\left(\frac{n}{2 \Delta t}\right)^{2}\right)\right)
\end{gathered}
$$

for $|n|$ and $|m| \leq N$ and where $A_{\alpha}$ is a constant.

However, both presented cases assumed that the Wigner distribution of $x(t)$ is zero outside an origin-centered circle of diameter equal to the sampling period [58]. Therefore, there might be several DFRFT matrices providing the same result within the accuracy of this approximation. Nevertheless, if the signal energy 
contained within this circle is approaching to the total signal energy, we know that all of these matrices provide more accurate results. In other words, signals can be recovered from their transforms only within some approximation errors [59], [60].

In order to alleviate some of the problems associated with the DFRFT proposed in [58], a new type of DFRFT, which is unitary, reversible, and flexible, was proposed in [56]. In addition, the closed-form analytic expression of this DFRFT can be obtained. Its performance is similar to the FRFT and can be efficiently calculated by FFT. Assuming that the samples of the input function, $x(t)$, and the output function $X_{\alpha}(u)$ of the FRFT are obtained by the interval $\Delta t, \Delta u$ as

$$
y(n)=x(n \Delta t) \quad Y_{\alpha}(m)=X_{\alpha}(m \Delta u)
$$

where $n=-N,-N+1, \ldots, N$ and $m=$ $-M,-M+1, \ldots, M$, then, the following two formulas of the DFRFT are in order:

$$
\begin{aligned}
& Y_{\alpha}(m)=\sqrt{\frac{\sin \alpha-j \cos \alpha}{2 M+1}} \\
& \times \exp \left(\frac{j}{2} \cot \alpha m^{2} \Delta u^{2}\right) \\
& \times \sum_{n=-N}^{N} \exp \left(-j \frac{2 \pi n m}{2 M+1}\right) \\
& \times \exp \left(\frac{j}{2} \cot \alpha n^{2} \Delta t^{2}\right) y(n)
\end{aligned}
$$

when $\sin \alpha>0(\alpha \in 2 D \pi+(0, \pi), D \in \mathbb{Z})$, and

$$
\begin{gathered}
Y_{\alpha}(m)=\sqrt{\frac{-\sin \alpha-j \cos \alpha}{2 M+1}} \\
\times \exp \left(\frac{j}{2} \cot \alpha m^{2} \Delta u^{2}\right) \\
\times \sum_{n=-N}^{N} \exp \left(j \frac{2 \pi n m}{2 M+1}\right) \\
\times \exp \left(\frac{j}{2} \cot \alpha n^{2} \Delta t^{2}\right) y(n)
\end{gathered}
$$

when $\sin \alpha<0(\alpha \in 2 D \pi+(-\pi, 0), D \in \mathbb{Z})$. Additionally, the constraints that $M \geq N$ $(2 N+1,2 M+1$ are, respectively, the number of points in the time and frequency domains) and

$$
\Delta u \Delta t=2 \pi|\sin \alpha| /(2 M+1)
$$

must also be satisfied. We note that when $M=N$ and $\alpha=\pi / 2(-\pi / 2)$, equations (44) and (45) become the DFT or IDFT. We also note that when $\alpha=D \pi(D \in \mathbb{Z})$, there is no proper choice for $\Delta u$ and $\Delta t$ that satisfies the inverse formula, and thus, we cannot use the above as the definition of the DFRFT. In fact, in these cases, we can just use the following definitions:

$$
\begin{aligned}
& Y_{\alpha}(m)=y(m) \text { when } \alpha=2 D \pi \\
& Y_{\alpha}(m)=y(-m) \text { when } \alpha=(2 D+1) \pi .
\end{aligned}
$$

The inverse DFRFT is simply a Hermitian forward DFRFT with $-\alpha$. This DFRFT is efficient to calculate and implement. Because there are two chirp multiplications and one FFT, the total number of the multiplication operations required is $2 P+(P / 2) \log _{2} P$, where $P=2 M+1$ is the length of the output. The authors also claimed that this DFRFT also has the lowest complexity among all types of DFRFT that still work similarly to the continuous FRFT [56]. However, it does not match the continuous FRFT and lacks many of the characteristics of the continuous FRFT. For example, it is difficult to filter out the chirp noise with this type of DFRFT [56].

\section{B. Linear combination-type DFRFT}

One of the first approaches for the DFRFT was proposed by Dickinson and Steiglitz in 1982 [61]. In their paper, the DFRFT was derived by using the linear combination of identity operation $\left(\mathbf{F}_{0}\right)$, discrete Fourier transform $\left(\mathbf{F}_{\pi / 2}\right)$, time inverse operation $\left(\mathbf{F}_{\pi}\right)$, and the inverse discrete Fourier transform $\left(\mathbf{F}_{3 \pi / 2}\right)$. In other words, the fractional matrix operator, $\mathbf{F}_{\alpha}$, for $0 \leq \alpha \leq \pi / 2$ was given as [61]:

$$
\mathbf{F}_{\alpha}=\sum_{k=0}^{3} \beta_{k} \mathbf{F}_{k \pi / 2}
$$

where

$$
\beta_{k}=\frac{1}{4} \sum_{l=1}^{4} \exp \left[j \pi l\left(\frac{2 \alpha}{\pi}-k\right) / 2\right]
$$


for $0 \leq k \leq 3$. In particular, it has been shown that the operator defined by (49) is unitary [62]. In other words,

$$
\begin{aligned}
\mathbf{F}_{\alpha}^{H} & =\mathbf{F}_{\alpha}^{-1}=\mathbf{F}_{-\alpha} \\
\mathbf{F}_{\alpha} \mathbf{F}_{-\alpha} & =\mathbf{I}
\end{aligned}
$$

Furthermore, the operator satisfies the angle additivity property [62]:

$$
\mathbf{F}_{\alpha_{1}} \mathbf{F}_{\alpha_{2}}=\mathbf{F}_{\alpha_{1}+\alpha_{2}}
$$

and angle multiciplity property [62]:

$$
\mathbf{F}_{\alpha}^{m}=\mathbf{F}_{m \alpha}
$$

It has also been noticed that the operator is periodic in the parameter $\alpha$ with a fundamental period $2 \pi[62]$.

This type of the DFRFT corresponds to a completely distinct definition of the fractional Fourier transform. However, the main problem is that the transform results will not match to the continuous FRFT [60]. In other words, it is not the discrete version of the continuous transform [63].

\section{DFRFT based on eigenvectors}

A possible approach for the DFRFT is based on searching the eigenvectors and eigenvalues of the DFT matrix and then computing the fractional power of the DFT matrix (e.g., [59], [60], [63], [64]). This type of the DFRFT was proposed to combat the issues associated with previous implementations such as a lack of unitarity and index additivity (e.g., [57] and [58]) and the fact that most of these provide a satisfactory approximation to the continuous transform. In particular, the DFRFT is based on the eigendecomposition of the DFT kernel matrix [60]. The transform kernel of the DFRFT can be defined as

$$
\begin{gathered}
\mathbf{F}_{2 \alpha / \pi}=\mathbf{U D}_{2 \alpha / \pi} \mathbf{U} \\
\mathbf{F}_{2 \alpha / \pi} \\
=\left\{\begin{array}{lc}
\sum_{k=0}^{N-1} \exp (-j k \alpha) & \text { for } \\
\times \mathbf{u}_{k} \mathbf{u}_{k}^{T} & N \text { odd } \\
\sum_{k=0}^{N-2} \exp (-j k \alpha) \mathbf{u}_{k} \mathbf{u}_{k}^{T} & \text { for } \\
+\exp (-j N \alpha) \mathbf{u}_{N} \mathbf{u}_{N}^{T} & N \text { even }
\end{array}\right.
\end{gathered}
$$

where $\mathbf{U}=\left[\begin{array}{llll}\mathbf{u}_{0} & \mathbf{u}_{1} & \ldots & \mathbf{u}_{N-1}\end{array}\right]$, when $N$ is odd, and $\mathbf{U}=\left[\begin{array}{lllll}\mathbf{u}_{0} & \mathbf{u}_{1} & \ldots & \mathbf{u}_{N-2} & \mathbf{u}_{N}\end{array}\right]$ when $N$ is even. $\mathbf{u}_{k}$ is the normalized eigenvector corresponding to the $k$ th order Hermite function and $\mathbf{D}_{2 \alpha / \pi}$ is defined as the following diagonal matrix :

$$
\begin{gathered}
\mathbf{D}_{2 \alpha / \pi}=\operatorname{diag}(\exp (-j 0), \exp (-j \alpha), \ldots, \\
\exp (-j \alpha(N-2)), \exp (-j \alpha(N-1)))
\end{gathered}
$$

for $N$ odd and

$$
\begin{gathered}
\mathbf{D}_{2 \alpha / \pi}=\operatorname{diag}(\exp (-j 0), \exp (-j \alpha), \ldots, \\
\exp (-j \alpha(N-2)), \exp (-j \alpha N))
\end{gathered}
$$

for $N$ even. In order to ensure orthogonality of the DFT Hermite eigenvectors $\left(\mathbf{u}_{k}\right)$, the Gram-Schmidt Algorithm (GSA) or the Orthogonal Procrustes Algorithm (OPA) can be used [60]. The GSA minimizes the errors between the samples of the Hermite functions and the orthogonal DFT Hermite eigenvectors. On the other hand, the OPA minimizes the total errors between those samples. It should be also pointed out that the main difference between the approach proposed in [60] and similar approaches proposed (e.g., [59], [63], [64]) is found in the obtained eigenvectors in previous contributions, in that they were just discrete Mathieu functions [60]. Although the Mathieu functions can converge to Hermite functions, the convergence for the eigenvectors obtained in previous approaches are not so fast for the high-order Hermite functions by the linear mean square error criterion [60]. Additionally, the authors investigated the relationship between the FRFT and the DFRFT and found that for a sampling period equal to $\sqrt{2 \pi / N}$, the DFRFT performs a circular rotation of the signal in the time-frequency plane [60]. However, the DFRFT becomes an elliptical rotation in the continuous time-frequency plane for sampling periods different from $\sqrt{2 \pi / N}$ [60]. Therefore, for these elliptical rotations an angle modification and a post-phase compensation in the DFRFT are required to obtain results similar to the continuous FRFT [60]. This approach has been extended to the socalled multiple-parameter discrete fractional Fourier transform (MPDFRFT) [65], [66]. In 
fact, the MPDFRFT maintains all of the desired properties and reduces to the DFRFT when all of its order parameters are the same.

A similar approach to [60] has been proposed in [67]. However, authors in [67] believe that the discrete time counterparts of the continuous time Hermite-Gaussians maintained the same properties, since these discrete time counterparts exhibited better approximations than the other proposed approaches [68]. In order to resolve this issue about the approximation of Hermite-Gaussian functions, a nearly tri-diagonal commuting matrix of the DFT and a corresponding version of the DFRFT was proposed in [69]. Most of the eigenvectors of this proposed nearly tridiagonal matrix result in a good approximation of the continuous Hermite-Gaussian functions by providing a smaller approximation error in comparison to previous approaches.

Using the findings presented in [60] and [67], methods for parallel and cascade computations of DFRFT are proposed in [70]. By this new method, the DFRFT of any angle can be computed by a linear combination of the DFRFTs with special angles [70]. The parallel method is suitable for the signal whose DFRFT in special angles are already known. The chirp signal detection is a common one, and the cascade method has a regular structure; therefore, it is very suitable for VLSI implementation.

However, it should be noted that these types of DFRFT's lack the fast computation algorithm and the eigenvectors cannot be written in a closed form.

\section{Other approaches}

The FRFT can also be realized by the quadratic phase transform (QPT) [71], [72], which is defined as

$$
\mathrm{QP}(\mu, \nu)=\int_{-\infty}^{+\infty} x(t) e^{-j \mu t-j \nu t^{2}} d t .
$$

Compared with (1), the FRFT can be computed by mapping variables $\mu$ and $\nu$ of the QPT onto $\alpha$ and $u$ of the FRFT. With this equivalence of implementation, a number of fast algorithms for the QPT can be used to compute the FRFT [71], [73]-[75]. Specifically, the fast algorithms proposed in [71] make use of the concept of decimation-in-time decomposition along the $\mu$ and $\nu$ domain. These algorithms can reduce the numbers of both complex multiplications and additions by a factor $2 \log _{2} N$, where $N$ is the number of samples in the time domain. By further exploiting the periodic and symmetric properties of the QPT and with the radix-2 decimation-in-frequency principle, a computationally more efficient algorithm was proposed in [73]. Recently, the above fast algorithms are also extended to real value sequences [75], which are useful in voice, audio, and image signal analysis.

Several other approaches for obtaining the DFRFT were proposed in the literature. Here, we only provide a brief overview of these techniques. For complete details, a reader should refer to these contributions. Using the group theory, authors in [76] proposed that the DFRFT can be obtained as the multiplication of DFT and the periodic chirps. This DFRFT satisfies the rotation property on the Wigner distribution, and the additivity and reversible property. However, this type of DFRFT can be derived only when the fractional order of the DFRFT equals some specified angles. As well, when the number of points is not prime, it will be very complicated to derive. Using Chirp-Z transforms, a fast numerical algorithm for the DRFT was proposed in [77]. This method allows free choice of resolutions in both fractional Fourier spaces, simultaneous data-peeping in any region, and easy implementation. Furthermore, the authors argued that their method is easier to implement in comparison with the method proposed in [61], while maintaining the same computational efficiency.

\section{Applications}

In this section, we review the practical applications of the FRFT and its discrete counterpart as a signal processing tool. Nevertheless, our literature review showed that applications are very scarce beyond optics. We anticipate additional publications regarding practical applications of the FRFT are bound to appear. For example, we foresee an increased number of applications of the FRFT based timefrequency representations in speech and music 
processing, biomedical signal processing, and mechanical vibrations analysis. Some problems stemming from such applications demand such advanced time-frequency representations (e.g., spectrogram, the Wigner distribution), since these classical time-frequency tools do not provide a framework sufficient for a comprehensive analysis [2].

It should be also mentioned that in this paper we devote more space to newer applications of the FRFT such as watermarking and communications. Other contributions (e.g., [1], [9]) described either more traditional applications such as filtering and signal recovery or only briefly covered different applications. Our goal is to emphasize that the FRFT is a valuable tool in many various applications.

\section{A. Filtering}

The idea of using the FRFT for fundamental signal processing procedures such filtering, estimation and restoration is particularly interesting for applications involving optical information processing [78], [79]. In several publications the concepts of filtering, estimation and restoration of signals in fractional domains were developed for these applications, revealing that under certain conditions one can improve upon the special cases of these operations in the conventional space and frequency domain. Furthermore, the FRFT can be applied to the problem of time-varying filtering of non-stationary, finite energy processes both in continuous-time and discrete-time frameworks [80]. Filtering in fractional Fourier domains may enable significant reduction of the mean square error in comparison with ordinary Fourier domain filtering. In particular, the optimum multiplicative filter function that minimizes the mean square error in the $\alpha$ th fractional Fourier domain was derived in [80]. Similarly, a novel fractional adaptive filtering scheme was introduced [81], and simulation results showed that adaptive filtering in the fractional domain is superior in comparison to its time domain counterparts.

\section{B. Watermarking}

The FRFT and its counterparts have not be fully exploited in the field of the multime- dia due to several plausible reasons. Firstly, several well established transforms are already used in the multimedia applications (e.g., DCT, DST, Walsh, DWT, DFT). Secondly, multimedia applications are commonly subject to some sort of standardization. Also, a relatively short history of the fast algorithms for the FRFT realization and a short period for the detailed study of the FRFT properties limit its wider usage in this area. The third reason is that this transform with a relatively long history in the other signal processing related disciplines, is still relatively unknown in the multimedia systems. However, this situation is gradually changing with more papers focusing on this transform. Here, it is worthwhile mentioning the application in the digital watermarking (e.g., [82]-[84]). The digital watermarking is a technique used for the copyright protection of digital multimedia data [85]. A desired ability for these techniques is a creation of a large number of watermarks that can be embedded in multimedia data without perceptible degradation of host data. Then, the FRFT domains offer more flexibility since it has been shown that the FRFT watermarks with various angles have small correlation. Usage of two angles in the 2D FRFT offers significant possibility to hide more different watermarks in images than the standard DFT or DCT-based domains.

This is illustrated with Figures 5 and 6 taken from [82]. In this figure the original Lena image used as a common test example is given in Figure 5(a) while the watermarked image is given in Figure 5(b). Watermark is embedded for angles $\alpha_{1}=\alpha_{2}=0.375 \pi$. Detection of watermarks for 1000 watermark keys is demonstrated in Figure 6(a) where upper line corresponds to detection in the watermarked image while lower line corresponds to detection in non-watermarked image. From spread between these two lines we can conclude that watermark detection is reliable. Figure $6(\mathrm{~b})$ demonstrates a situation when a search with the proper watermark key is performed around proper angles. It can be seen that the watermark can be detected only if the angle under which the watermark is embedded is known. This is a quite important fact and it helps 


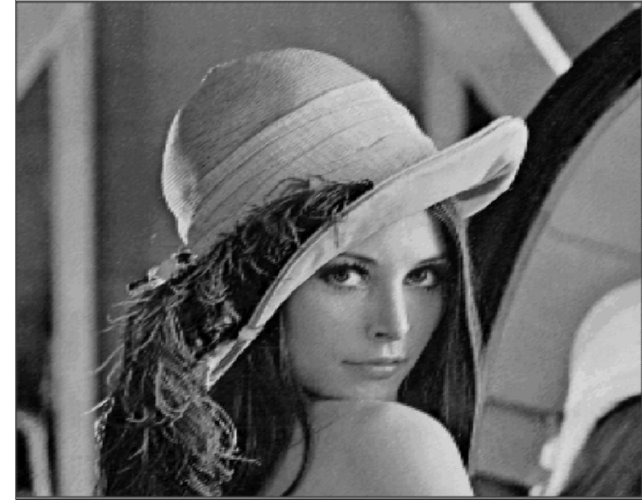

a)

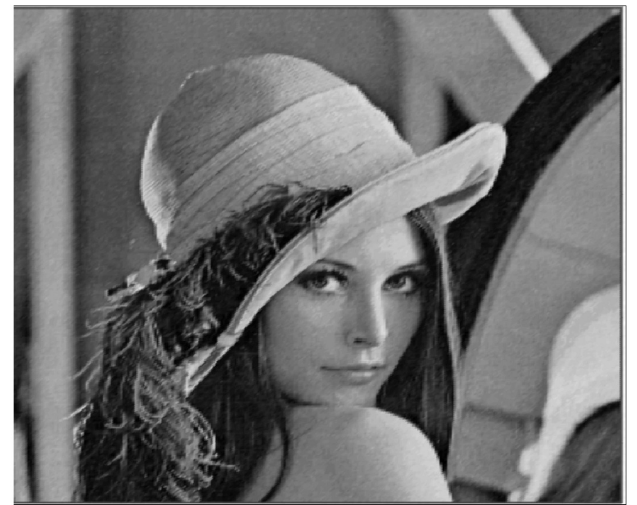

b)

Fig. 5. Watermarking using the FRFT: (a) original image; (b) watermarked image.

in increasing the number of watermarks that can be embedded within multimedia data. A practically useful scheme for the FRFT based watermarking is recently proposed in [86]. In particular, the impact of the noise and other errors is severely reduced on the FRFT coefficients by pre-filtering these coefficients yielding a scheme that is more robust to various attacks.

Similarly, a watermarking scheme in the space/spatial-frequency domain has been proposed in [87]. This watermarking technique uses 2D chirp signals that are well concentrated in the joint space/spatial-frequency domain, but not in the space or spatial-frequency domain only. Therefore, this concept of watermarking provides results robust to the standard filtering, since watermarks are concentrated in the joint space/spatial-frequency domain [87].

Both of these techniques are closely monitored and followed by numerous researchers [88]-[97]. The main obstacle in further applications of these techniques is the complexity that can be reduced with various previously described fast realization strategies. In addition, the availability of the FRFT fast realizations will lead to numerous implementations of the FRFT and related transforms in multimedia.

\section{Radar applications}

A couple of techniques related to the FRFT have been recently used for focusing SAR/ISAR images (e.g., [98], [99]). This technique is commonly referred as the LPFT in the related literature. Two forms of the adaptive LPFT-based technique for focusing ISAR images are proposed in [100]. The focusing is performed without assuming any particular model of a target's motion. Therefore, these versions of the LPFT can be applied for any realistic motion. The first technique is based on knowing that, for monocomponent and multicomponent signals with similar chirp-rates, a single chirp-rate parameter can be estimated for all components. The ISAR image is then focused by using the estimated value of signal parameters obtained through the LPFT calculation. For multicomponent signals with different chirp-rates, a sum of the weighted LPFT is used. If the signal's components have significantly different chirp-rates, the estimation of these parameters should be performed separately for each component. Moreover, the obtained estimate is additionally refined by combining estimations obtained for close reflectors. For targets with very complex motion patterns, segmentation of the radar image in regions-of-interests is performed. The adaptive LPFT is calculated for each region in order to form focused ISAR image. Adap- 


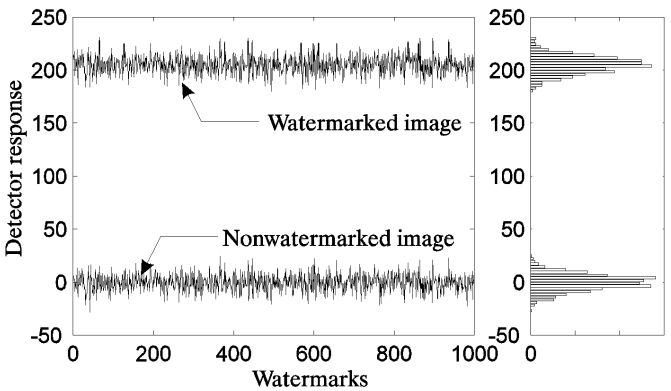

a)

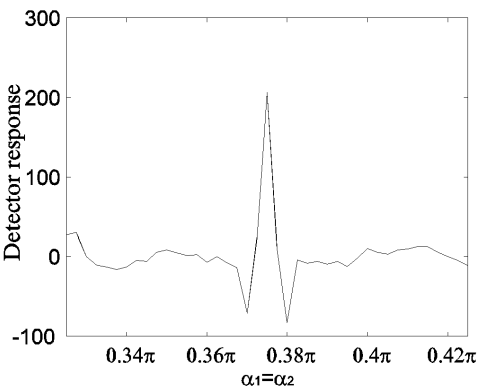

b)

Fig. 6. Analysis of the detector: (a) statistical analysis of detecting watermark signal in watermarked and nonwatermarked image; (b) detection of watermark signal using different transformation angles.

tive parameters are obtained by using a simple concentration measure. Here, we demonstrate test image of the B727 plane given on Figure 7 (a) (the figure is taken from [100]). Focusing of the radar image is performed for each cross-range parameter and the focused image is given in Figure 7(b). Estimated chirp-rate parameters (angles in the FRFT terminology) are depicted with dashed lines in Figure 7(c). Additional improvements have been achieved when these chirp-rates are filtered with median filter (thick line). Then, the radar images focused with the filtered chirp-rate as depicted in Figure 7(d).

A similar approach is proposed in [101], but the chirp-rate is estimated by using contrast of the LPFT-based ISAR image. Moreover, a modulus of the target effective rotation vector is calculated from the obtained estimation. A quantitative analysis of the signal-to-noise ratio (SNR) for the LPFT applied in the ISAR imaging is presented in [102]. It has been shown that the LPFT-based methods for focusing ISAR images can achieve a significantly higher output SNR than those based on the STFT.

Focusing of the SAR images is usually performed by estimating parameters of a received signal. A technique for applying the product higher-order ambiguity function (PHAF) for estimating parameters of the radar signal and focusing SAR images is proposed in [103]. Moving target focusing can be performed by this technique for some specific scenarios. More precisely, the obtained SAR im- age will be focused only when one target exists in a range bin, or when all targets in one range bin have similar motion parameters. Otherwise, this technique fails to achieve high concentration of each target simultaneously. In order to overcome this drawback, an algorithm for separating signal components corresponding to targets with different motion parameters is applied in [104] where the LPFT-based technique is used for focusing each component. The drawback of the LPFT-based technique, applied in this manner, is high computational complexity needed for selection of chirp-rate that produces the best concentration. Therefore, an algorithm for the automated selection of phase parameters used for the LPFT calculation is proposed in [105]. In this algorithm, an adaptive set of chirp-rates is formed for each unfocused target. When one or more unfocused targets are detected in a range, the PHAF is evaluated and the position of its maximum is used for the coarse estimation of chirprate. Further improvement of the obtained estimate is performed by using a fine search in the region around the chirp-rate obtained from the coarse search. The number of chirprates in the fine search stage is rather small (usually not larger than several tens) which yields a significant decrease in the computational complexity with respect to the technique proposed in [104] (which requires hundreds or thousands). In addition, a procedure for the third-order phase compensation is applied in the proposed algorithm without a significant increase of the calculation burden. In 

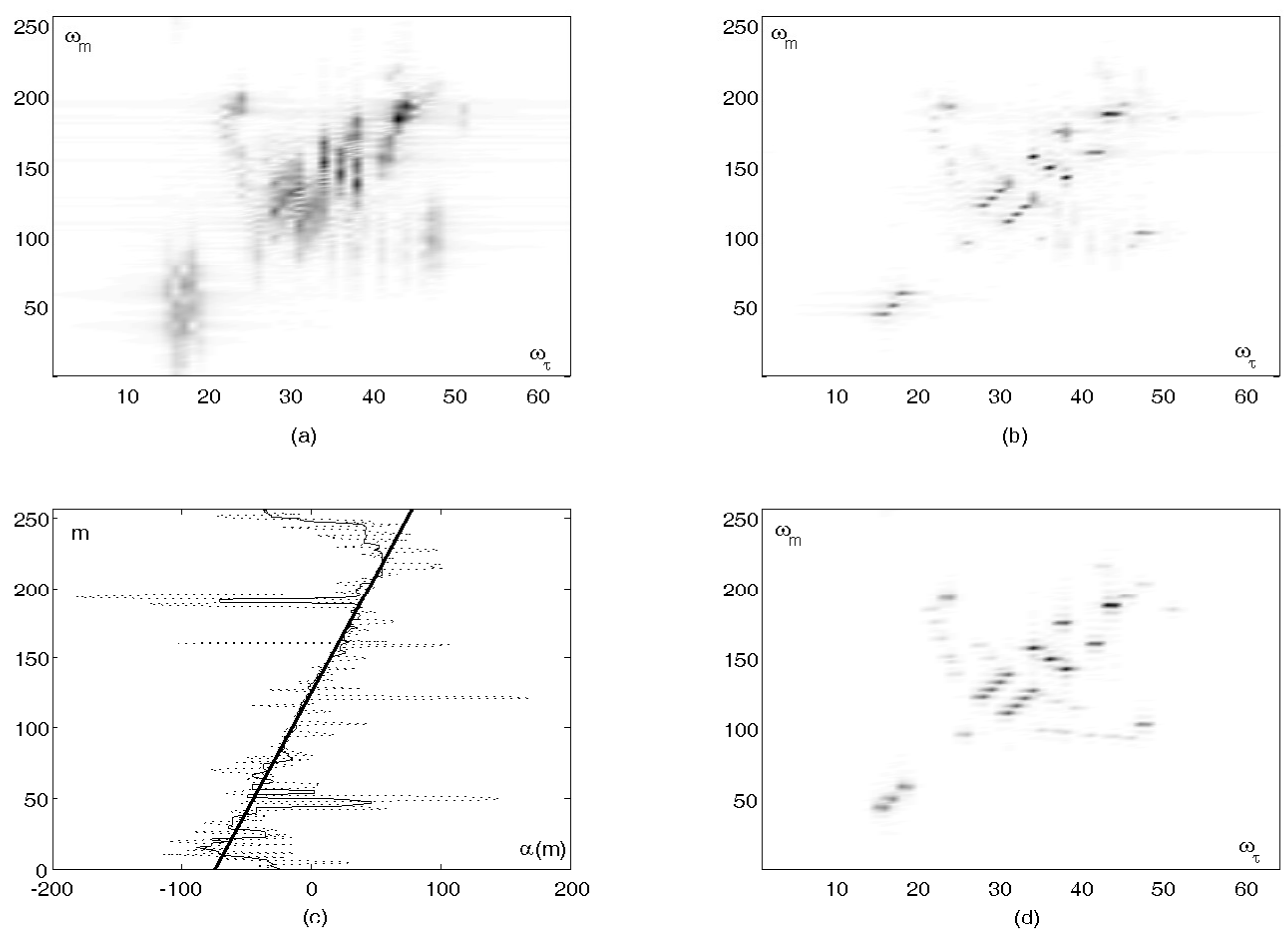

Fig. 7. B727 radar image: (a) results obtained by the FT-based method; (b) adaptive LPFT method; (c) adaptive chirp rate (dotted line), filtered adaptive chirp rate (light solid line), linear interpolation of filtered data (bold solid line); (d) adaptive LPFT with interpolated data. $\omega_{\tau}$ and $\omega_{m}$ represent the range and the cross-range, respectively.

[104], [105], [106] it has been shown that the LPFT-based methods for focusing SAR images are more robust to the additive noise influence than the STFT radar imaging techniques.

Here, we have borrowed figure from [105] where the SAR image of several moving and stationary targets is shown (Figure 8(a)). It can be seen that the moving targets have seriously spread components. Alternative tools such as a technique called the S-method [107] are unable to separate overlapping targets (Figure 8(b)). The LPFT technique with predefined set of the chirp-rates gives significantly improved results as shown in Figure 8(c). However, the procedure proposed in [105] based on the LPFT with automatic selection of the chirp-rate parameters, produces highly focusing of all considered targets (Figure 8(d)).

\section{Communications}

Traditional multicarrier systems (MC) are designed with the DFT based schemes. The main representative of these techniques is the orthogonal frequency-division multiplexing (OFDM). However, for doubly-selective channels (channels with selectivity in both time and frequency) this technique produces non-satisfactory results. Martone in 2001 published a corner-stone paper where the discrete FRFT is used instead of the DFT in multicarrier systems [108]. For channels with fast variations with available the line-of-sight component (usually associated with mobile stations mounted on high speed carriers and/or non-urban environments) this scheme significantly outperforms DFT-based counterparts. Performance of this technique is significantly improved since the time-frequency plane can be adjusted (rotated) in a way to compensate undesired modulation of the signals introduced by high velocity of participants and/or by multipath component shifted from the line-of-sight components. This technique is generalized for general linear canonical form of the transform 

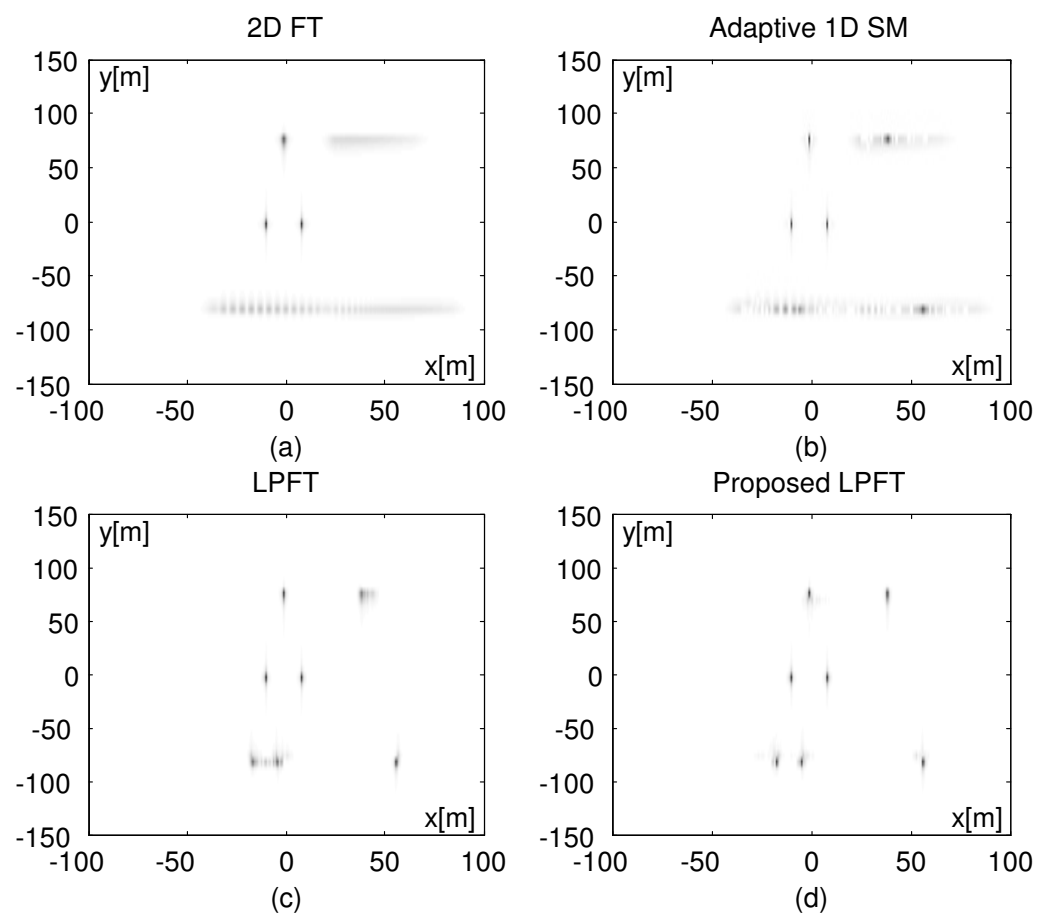

Fig. 8. Simulated SAR image of seven target points obtained by using: (a) 2D FT; (b) adaptive S-method; (c) LPFT with predefined set of chirp rates; and (d) proposed LPFT.

(affine Fourier transform - AFT) in [109]. This form has shown an improved flexibility with respect to the FRFT-based scheme. These chirp based modulations and associated AFT schemes have been identified as a suitable basis for multicarrier communications such as aeronautical and satellite [110]. Table IV provides a comparison between the AFT-MC technique and the standard OFDM for four typical scenaria that can be observed in the case of the aeronautical channels. The best results for the AFT-MC techniques are observed for en-route scenario while in the worst case for the parking scenario, the AFT-based technique behaves the same as the OFDM. Then, in our opinion the AFT technique are important candidates for novel digital standards for the aeronautical communications with solved problem of the fast realizations of the AFT (FRFT).

Similarly, other contributions discussed the application of the FRFT in communication systems. In a very recent publication (e.g., [111]), a minimum mean squared error re- ceiver based on the FRFT for MIMO systems with space time processing over Rayleigh faded channels was presented. The numerical analysis of the proposed receiver showed improved performance; outperforming the simple minimum mean squared error receiver in Rayleigh faded channel. Furthermore, chirp modulation spread spectrum based on the FRFT was recently proposed for demodulation of multiple access systems [112]. The numerical analysis showed that the FRFT based receiver is more flexible and efficient system for multiple access by reducing the designing complexity of the system. The authors also argued that receivers based on the FRFT are more sensitive than earlier coherent receivers for chirp signals.

\section{E. Compression}

An interesting application of the FRFT to compression can be found in [113]. In particular, the contribution dealt with image compression and found that even though the pre- 
TABLE IV

A Comparison of two techniques.

\begin{tabular}{c|c|l|l|l}
\hline \hline Scenario & En-route & Arrival/Takeoff & Taxi & Parking \\
\hline AFT-MC vs. OFDM & Significantly better & Slightly better & Slightly better & Equal \\
\hline
\end{tabular}

sented method yielded inferior results in comparison to commonly available compression algorithms, there is still a room for improvement. The authors argued that their method is very basic and further improvements can be achieved by combining with other techniques. The concept of compression using the FRFT has been further extended in [114], where authors proposed a scheme for signal compression based on the combination of DFRFT and set partitioning in hierarchical tree (SPIHT). The application of the scheme to different types of signals demonstrated a significant reduction in bits leading to high signal compression ratio. The results were further compared with those obtained with the discrete cosine transform. Hence, the DFRFT is shown to be more suitable for compression than the DCT, especially in terms of quality of reconstructed signal and the percentage root-mean-square difference. There is no need to encode the error signal and send along with the encoded DFRFT coefficients, as the dynamic range of error signal is too small.

\section{F. Pattern recognition}

Given that the FRFT provides an extra parameter (e.g., the rotation angle) in comparison with the ordinary Fourier transform, the FRFT also provides additional degrees of freedom in pattern recognition systems. For example, the FRFT was used as a pre-processor for a neural network [115]. The use of fractional based pre-processing resulted in an improved performance comparing to both no preprocessing and ordinary Fourier transform preprocessing. Also, the fractional based preprocessing resulted in a substantial reduction of classification and localization error. While use of the fractional Fourier transform increases the cost of the training procedure, the improvements achieved with its use come at no additional routine operating cost.
Furthermore, it has been also shown that pattern recognition methods based on matched filtering can be generalized by replacing the standard Fourier operations by fractional Fourier operations [116]. However, these systems become time (or space) varying systems.

\section{G. Cryptography}

The FRFT can also be used in the field of cryptography [117]-[121]. For example, a 2004 US patent invented a cryptographic approach [117]. At the encryption side, a user first selects at least four parameters including an angle of rotation, a time exponent, a phase, and a sampling rate as the encryption key. Then, more than one modified FRFT kernels corresponding to the encryption key are selected and multiplied with the input signal. On the other hand, a reverse procedure with respect to the encryption process is used for decryption.

The modified FRFT (e.g., [118]) along with the double random phase encoding technique has been successfully applied for encrypting digital data. Specifically, using the double random phase encoding in the multiple-parameter FRFT domain, this encryption method enhances data security because the order parameters of the modified FRFT can be exploited as extra keys for decryption. However, this encryption scheme has shown to be linear [119]. Hence, it is insecure because a known plaintext attack can break this scheme, which is equivalent to solving a set of linear equations [119]. It is also shown that the current standard algorithms such as AES [122] outperform the above system in terms of both encryption speed, bandwidth, and storage requirements.

Recently, a random discrete FRFT, proposed in [123], exhibits an important feature that the magnitude and phase of its transform output are both random. This random dis- 
crete FRFT is also first applied to the image encryption to show its potentials.

\section{H. Fractal signal processing}

The FRFT also found its application in processing of fractal signals. For example, the FRFT was used for the determination of the main parameters of fractals in [124]. In [125], a FRFT based estimation method was introduced to analyze the long range dependence in time series. In particular, the FRFT was used for estimation of the Hurst exponent. The results have shown that the FRFT estimator can achieve a reliable estimation of the Hurst exponent when compared to some other existing estimation methods, such as wavelet-based method and a global estimator based on dispersional analysis.

\section{Other applications}

The FRFT can be useful in terms of differential equations [7], [126], especially for solving these equations. For example, the FRFT of a function $x(t)$ can be considered as a solution of a differential equation, where $x(t)$ can be considered as the initial condition of the equation. In particular, Namias solved several Shrodinger equations using this assumption [7]. Further examples can be found in [1], [7], [126]. The FRFT can be also shown to be a particular case of the evolution operators [127]. Further details about the application of the FRFT to differential equations can be found in [128].

An application of the FRFT to computer tomography was also recently discussed [129]. In particular, a two-dimensional FRFT has been used to characterize the effect of scattering [129].

The FRFT has also been applied to transient motor current signature analysis (TMCSA) [130], due to the shortcomings of Fourier transform for such an analysis. This paper also proposed the optimization of the FRFT to generate a spectrum where the frequencyvarying fault harmonics appear as single spectral lines and, therefore, facilitate the diagnostic process.

\section{Conclusion}

The FRFT is a powerful mathematical transform that generalizes the ordinary Fourier transform through the order parameter $\alpha$. As such, it has been rediscovered in the literature several times. In this paper, we provided an overview of the FRFT from the signal processing point of view. In particular, our goal was to attract signal processing practitioners to this mathematically eloquent concept and depict its advantages. In order to do so, the paper is thematically divided into three topics. In the first part, we presented the definition of the FRFT and tied it to other mathematical representations. The second part of the paper covered various practical realizations of the FRFT, while the practical applications of the FRFT were discussed in the third part of the paper. The conclusions stemming from each part were as follows:

- Besides its ties to the ordinary Fourier transform, the FRFT can be further related to various time-frequency transforms. In particular, we showed the direct relationship between the AF, the WD and the FRFT. This relationship enables us to relate the FRFT to a wide class of time-frequency transforms. Furthermore, we showed that the FRFT can be considered as a special case of the second-order LPFT and LPP. Also, the FRFT can be considered as the special case of the LCT. Such a relationship provides a direct generalization link between the FRFT and many higher-order/affine mathematical transformations. In particular, we outlined the connection between the FRFT and L-WD for the first time in the literature. Future contributions in this theme will include a further understanding of the FRFT and its ties to other mathematical transforms.

- The digital realizations of the FRFT can be divided into three major streams. One stream is represented through direct sampling of the FRFT. It is the least complicated approach, but these discrete realizations could lose many important properties of the FRFT. Furthermore, the kernel associated with the FRFT can introduce very fast oscillations that require excessively large sampling rates. A second stream relies on a linear combination of ordinary Fourier operators raised to different 
powers. Nevertheless, these realizations often produce an output that does not match the output of the continuous FRFT. The third stream approaches the discrete realizations based on the idea of an eigenvalue decomposition. The realizations obtained through this stream tend to closely resemble the representations obtained by the continuous FRFT. However, we should point out that the major drawbacks of this approach are that they cannot be written in a closed form and might have computational costs. Lastly, we also outlined other approaches that appeared in the literature. We specifically suggested that potential realizations of the FRFT could be achieved with the so-called quadratic phase transform. Anticipated contributions in this field need to deal with issues associated with the current schemes.

- The number of publications discussing practical applications of the FRFT has been steadily rising. In particular, we have witnessed a strong expansion of the FRFT in several fields, including watermarking, radar applications, and wireless communications. Furthermore, we also have the FRFT present in other fields as well (e.g., fractal signal processing, pattern recognition, filtering). The main advantage is that the FRFT-based schemes increase processing accuracy. For example, a group of authors described an adaptive filtering scheme in a recent contribution. The presented results showed a superior performance in comparison to its time domain counterparts. Similar trends have been observed in multimedia signal processing, where the FRFT has been used for watermarking. In particular, the watermark could be detected only if the angle under which the watermark was embedded was known. This enabled us to increase the number of watermarks embedded within multimedia data. From these past contributions, we expect an expansion of FRFT-based methods in different applications to dominate the future contributions. In particular, we expect to see increased application of the FRFT based time-frequency representations in speech and music processing, biomedical signal processing and mechanical vibrations analysis. Problems stemming from such applications require the employment of such advanced time-frequency transforms.

Overall, the paper provided a compact summary of the recent contributions regarding the FRFT (for your convenience, Table V contains reference sorted according to their topics). The FRFT is a very powerful tool and has been applied to many fields. Further research and applications of existing schemes will increase in the near future.

\section{ACKNOWLEDGMENTS}

The work of Igor Djurović is supported in part by the Ministry of Science and Education of Montenegro. The authors would like to thank Vesna Popović from the University of Montenegro, Djuro Stojanović from Crnogorski Telekom and $\mathrm{Pu}$ Wang from the Stevens Institute of Technology for shaping up some sections of this manuscript. Last, but not least, the authors would like to thank the reviewers whose comments have helped them to improve the technical quality and the presentation of the manuscript.

\section{REFERENCES}

[1] H. M. Ozaktas, Z. Zalevsky, and M. A. Kutay, The fractional Fourier transform with applications in optics and signal processing. Chichester, New York, USA: John Wiley, 2001.

[2] E. Sejdić, I. Djurović, and J. Jiang, "Timefrequency feature representation using energy concentration: An overview of recent advances," Digital Signal Processing, vol. 19, no. 1, pp. 153183, Jan. 2009.

[3] E. Sejdić, I. Djurović, J. Jiang, and LJ. Stankovic, Time-Frequency Based Feature Extraction and Classification: Considering Energy Concentration as a Feature Using Stockwell Transform and Related Approaches. Saarbrucken, Germany: VDM Verlag Publishing, 2009.

[4] N. Wiener, "Hermitian polynomials and Fourier analysis," Journal of Mathematical Physics (MIT), vol. 8, pp. 70-73, 1929.

[5] E. U. Condon, "Immersion of the Fourier transform in a continuous group of functional transformations," Proceedings of the National Academy of Sciences of the United States of America, vol. 23, no. 3, pp. 158-164, Mar. 1937.

[6] N. G. De Bruijn, "A theory of generalized functions, with applications to Wigner distribution and Weyl correspondence," Nieuw Archief voor Wiskunde, vol. 21, pp. 205-280, 1973.

[7] V. Namias, "The fractional order Fourier transform and its application to quantum mechanics," IMA Journal of Applied Mathematics, vol. 25, no. 3, pp. 241-265, Mar. 1980. 
TABLE V

REFERENCES SORTED ACCORDING TO THEIR TOPICS.

\begin{tabular}{l|l|l}
\hline \hline \multicolumn{2}{c|}{ Topics } & References \\
\hline Theoretical contributions & Introductory and review contributions & {$[1],[4]-[15]$} \\
& FRFT and time-frequency representations & {$[2],[3],[16]-[44]$} \\
& FRFT and linear canonical transform & {$[1],[6],[45],[46]$} \\
& DFRFT through sampling of FRFT & {$[14],[50]-[58]$} \\
& Linear combination-type DFRFT & {$[61],[62]$} \\
& DFRFT based on eigenvectors & {$[59],[60],[63]-[70]$} \\
& Other approaches for DFRFT & {$[71]-[77]$} \\
\hline Applications & Filtering & {$[78]-[81]$} \\
& Watermarking & {$[82]-[84],[86]-[97]$} \\
& Radar applications & {$[98]-[106]$} \\
& Communications & {$[108]-[112]$} \\
& Compression & {$[113],[114]$} \\
& Pattern recognition & {$[115],[116]$} \\
& Cryptography & {$[117]-[123]$} \\
& Fractal signal processing & {$[124],[125]$} \\
& Other application & {$[7],[126]-[130]$} \\
\hline
\end{tabular}

[8] V. A. Narayanan and K. M. M. Prabhu, "The fractional Fourier transform: theory, implementation and error analysis," Microprocessors and Microsystems, vol. 27 , no. 10, pp. 511-521, Nov. 2003.

[9] A. Bultheel and H. Martínez-Sulbaran, "Recent developments in the theory of the fractional Fourier and linear canonical transforms," The Bulletin of the Belgian Mathematical Society Simon Stevin, vol. 13, no. 5, pp. 971-1005, Jan. 2007.

[10] D. Mendlovic and H. M. Ozaktas, "Fractional Fourier transforms and their optical implementation: part I," Journal of the Optical Society of America A: Optics and Image Science, and Vision, vol. 10, no. 9, pp. 1875-1881, Sep. 1993.

[11] _ _ "Fractional Fourier transforms and their optical implementation: part II," Journal of the Optical Society of America A: Optics and Image Science, and Vision, vol. 10, no. 12, pp. 2522 2531, Dec. 1993.

[12] L. B. Almeida, "The fractional Fourier transform and time-frequency representations," IEEE Transactions on Signal Processing, vol. 42, no. 11, pp. 3084-3091, Nov. 1994.

[13] G. Cariolaro, T. Erseghe, P. Kraniauskas, and N. Laurenti, "A unified framework for the fractional Fourier transform," IEEE Transactions on Signal Processing, vol. 46, no. 12, pp. 32063219, Dec. 1998.

[14] A. I. Zayed, "On the relationship between the Fourier and fractional Fourier transforms," IEEE Signal Processing Letters, vol. 3, no. 12 , pp. 310-311, Dec. 1996.

[15] L. B. Almeida, "Product and convolution theorems for the fractional Fourier transform," IEEE Signal Processing Letters, vol. 4, no. 1, pp. 1517, Jan. 1997.
[16] LJ. Stanković and I. Djurović, "Relationship between the ambiguity function coordinate transformations and the fractional Fourier transform," Annales des Telecommunications, vol. 53, no. $7 / 8$, pp. 316-319, Jul. 1998.

[17] T. Alieva and M. J. Bastiaans, "On fractional Fourier transform moments," IEEE Signal Processing Letters, vol. 7, no. 11, pp. 320323, Nov. 2000

[18] B. Ristic and B. Boashash, "Kernel design for time-frequency signal analysis using the Radon transform," IEEE Transactions on Signal Processing, vol. 41, no. 5, pp. 1996-2008, May 1993.

[19] H. M. Ozaktas, N. Erkaya, and M. A. Kutay, "Effecct of fractional Fourier transformation on time-frequency distributions belonging to the Cohen class," IEEE Signal Processing Letters, vol. 3, no. 2, pp. 40-41, Feb. 1996.

[20] M. J. Bastiaans, T. Alieva, and LJ. Stanković, "On rotated time-frequency kernels," IEEE Signal Processing Letters, vol. 9, no. 11, pp. 378381, Nov. 2002

[21] LJ. Stanković, T. Alieva, and M. J. Bastiaans, "Time-frequency signal analysis based on the windowed fractional Fourier transform," Signal Processing, vol. 83, no. 11, pp. 2459-2468, Nov. 2003.

[22] A. W. Lohmann, "Image rotation, Wigner rotation, and the fractional Fourier transform," Journal of the Optical Society of America A: Optics, Image Science, and Vision, vol. 10, no. 10, pp. 2181-2186, Oct. 1993.

[23] D. Mendlovic, Z. Zalevsky, R. G. Dorsch, Y. Bitran, A. W. Lohmann, and H. M. Ozaktas, "New signal representation based on the fractional Fourier transform: definitions," Journal of the Optical Society of America A: Optics and 
Image Science, and Vision, vol. 12, no. 11, pp. 2424-2431, Nov. 1995.

[24] D. Mustard, "The fractional Fourier transform and the Wigner distribution," The Journal of the Australian Mathematical Society - Series B - Applied Mathematics, vol. 38, no. 2, pp. 209219, Oct. 1996.

[25] S.-C. Pei and J.-J. Ding, "Relations between fractional operations and time-frequency distributions, and their applications," IEEE Transactions on Signal Processing, vol. 49, no. 8, pp. 1638-1655, Aug. 2001

[26] J. Wood and D. Barry, "Radon transformation of time-frequency distributions for analysis of multicomponent signals," IEEE Transactions on Signal Processing, vol. 42, no. 11, pp. 3166-3177, Nov. 1994.

[27] A. W. Lohmann and B. H. Soffer, "Relationships between the Radon-Wigner and fractional Fourier transforms," Journal of the Optical Society of America A: Optics, Image Science, and Vision, vol. 11, no. 6, pp. 1798-1801, Jun. 1994

[28] V. Katkovnik, "A new form of the Fourier transform for time-varying frequency estimation," Signal Processing, vol. 47, no. 2, pp. 187-200, Nov. 1995.

[29] _ - "Discrete-time local polynomial approximation of the instantaneous frequency," IEEE Transactions on Signal Processing, vol. 46, no. 10, pp. 2626-2637, Oct. 1998.

[30] S.-C. Pei and J.-J. Ding, "Relations between Gabor transforms and fractional Fourier transforms and their applications for signal processing," IEEE Transactions on Signal Processing, vol. 55 , no. 10 , pp. 4839-4850, Oct. 2007.

[31] H. M. Ozaktas, B. Barshan, D. Mendlovic, and L. Onural, "Convolution, filtering, and multiplexing in fractional Fourier domains and their relation to chirp and wavelet transforms," Journal of the Optical Society of America A: Optics and Image Science, and Vision, vol. 11, no. 2, pp. 547-559, Feb. 1994.

[32] S.-Y. Lee and H. H. Szu, "Fractional Fourier transforms, wavelet transforms, and adaptive neural networks," Optical Engineering, vol. 33, no. 7, pp. 2326-2330, Jul. 1994.

[33] C. Capus and K. Brown, "Fractional Fourier transform of the Gaussian and fractional domain signal support," IEE Proceedings - Vision, Image, and Signal Processing, vol. 150, no. 2, pp. 99-106, Apr. 2003.

[34] LJ. Stanković, T. Alieva, and M. J. Bastiaans, "Fractional-Fourier-domain weighted Wigner distribution," in Proc. of the 11th IEEE Signal Processing Workshop on Statistical Signal Processing, Singapore, Aug. 6-8, 2001, pp. 321324.

[35] A. Akan and L. Chaparro, "Discrete rotational Gabor transform," in Proc. of IEEE-SP International Symposium on Time-Frequency and Time-Scale Analysis, Paris, France, Jun. 18-21, 1996, pp. $169-172$.

[36] A. Akan, V. Shakhmurov, and Y. Cekic, "A fractional Gabor transform," in Proc. of IEEE International Conference on Acoustics, Speech, and Signal Processing (ICASSP 1994), vol. 6, Adelaide, SA, Australia, Apr. 19-22, 1994.
[37] A. Akan and Y. Çekiç, "A fractional Gabor expansion," Journal of the Franklin Institute, vol. 340, no. 5, pp. 391-397, Nov. 2003.

[38] Y. Zhang, B.-Y. Gu, B.-Z. Dong, G.-Z. Yang, H. Ren, X. Zhang, and S. Liu, "Fractional Gabor transform," Optics Letters, vol. 22, no. 21, pp. 1583-1585, Nov. 1997.

[39] F. Zhang, G. Bi, and Y. Q. Chen, "Tomography time-frequency transform," IEEE Transactions on Signal Processing, vol. 50, no. 6, pp. 12891297, Jun. 2002.

[40] X.-G. Xia, Y. Owechko, B. H. Soffer, and R. M. Matic, "On generalized-marginal time-frequency distributions," IEEE Transactions on Signal Processing, vol. 44 , no. 11 , pp. 2882-2886, Nov. 1996.

[41] S. Shinde and V. M. Gadre, "An uncertainty principle for real signals in the fractional Fourier transform domain," IEEE Transactions on Signal Processing, vol. 49, no. 11, pp. 2545-2548, Nov. 2001.

[42] X. Guanlei, W. Xiaotong, and X. Xiaogang, "The logarithmic, Heisenberg's and short-time uncertainty principles associated with fractional Fourier transform," Signal Processing, vol. 89, no. 3, pp. 339-343, Mar. 2009.

[43] _ _ "Three uncertainty relations for real signals associated with linear canonical transform," IET Signal Processing, vol. 3, no. 1, pp. 85-92, Jan. 2009.

[44] —-, "Generalized entropic uncertainty principle on fractional Fourier transform," Signal Processing, vol. 89 , no. 12 , pp. 2692-2697, Dec. 2009.

[45] S.-C. Pei and J.-J. Ding, "Eigenfunctions of linear canonical transform," IEEE Transactions on Signal Processing, vol. 50, no. 1, pp. 11-26, Jan. 2002.

[46] —-, "Simplified fractional Fourier transforms," Journal of the Optical Society of America A. Optics and Image Science, and Vision, vol. 17, no. 12 , pp. 2355-2367, Dec. 2000.

[47] LJ. Stanković, "A multitime definition of the Wigner higher order distribution: L-Wigner distribution," IEEE Signal Processing Letters, vol. 1, no. 7, pp. 106-109, Jul. 1994.

[48] B. Barkat and B. Boashash, "Design of higher order polynomial Wigner-Ville distributions," IEEE Transactions on Signal Processing, vol. 47 , no. 9, pp. 2608-2611, Sep. 1999.

[49] F. J. Marinho and L. M. Bernardo, "Numerical calculation of fractional Fourier transforms with a single fast-Fourier-transform algorithm," Journal of the Optical Society of America A: Optics, Image Science and Vision, vol. 15, no. 8, pp. 2111-2116, Aug. 1998.

[50] X.-G. Xia, "On bandlimited signals with fractional Fourier transform," IEEE Signal Processing Letters, vol. 3, no. 3, pp. 72-74, Mar. 1996.

[51] A. I. Zayed and A. G. Garcia, "New sampling formulae for the fractional Fourier transform," Signal Processing, vol. 77, no. 1, pp. 111-114, Aug. 1999.

[52] T. Erseghe, P. Kraniauskas, and G. Carioraro, "Unified fractional Fourier transform and sampling theorem," IEEE Transactions on Signal 
Processing, vol. 47, no. 12, pp. 3419-3423, Dec. 1999.

[53] C. Candan and H. M. Ozaktas, "Sampling and series expansion theorems for fractional Fourier and other transforms," Signal Processing, vol. 83, no. 11, pp. 2455-2457, nov 2003.

[54] K. K. Sharma and S. D. Joshi, "Fractional Fourier transform of bandlimited periodic signals and its sampling theorems," Optics Communications, vol. 256 , no. 4-6, pp. 272-278, Dec. 2005.

[55] R. Tao, B. Deng, W.-Q. Zhang, and Y. Wang, "Sampling and sampling rate conversion of band limited signals in the fractional Fourier transform domain," IEEE Transactions on Signal Processing, vol. 56, no. 1, pp. 158-171, Jan. 2008.

[56] S.-C. Pei and J.-J. Ding, "Closed-form discrete fractional and affine Fourier transforms," IEEE Transactions on Signal Processing, vol. 48, no. 5, pp. 1338-1353, May 2000.

[57] O. Arikan, M. A. Kutay, H. M. Ozaktas, and O. K. Akdemir, "The discrete fractional Fourier transformation," in Proceedings of the IEEE-SP International Symposium on Time-Frequency and Time-Scale Analysis, Jun. 18-21, 1996, pp. 205-207.

[58] H. M. Ozaktas, O. Arikan, M. A. Kutay, and G. Bozdagt, "Digital computation of the fractional Fourier transform," IEEE Transactions on Signal Processing, vol. 44, no. 9, pp. 21412150, Sep 1996

[59] S.-C. Pei and M.-H. Yeh, "Improved discrete fractional Fourier transform." Optics Letters, vol. 22, no. 14, pp. 1047-1049, Jul. 1997.

[60] S.-C. Pei, M. H. Yeh, and C. C. Tseng, "Discrete fractional Fourier transform based on orthogonal projections," IEEE Transactions on Signal Processing, vol. 47, no. 5, pp. 1335-1348, May 1999.

[61] B. W. Dickinson and K. Steiglitz, "Eigenvectors and functions of the discrete Fourier transform," IEEE Transactions on Acoustics, Speech, and Signal Processing, vol. 30, no. 1, pp. 25-31, Feb. 1982.

[62] B. Santhanam and J. H. McClellan, "The discrete rotational Fourier transform," IEEE Transactions on Signal Processing, vol. 44, no. 4, pp. 994-998, Apr 1996

[63] S.-C. Pei, C.-C. Tseng, M.-H. Yeh, and J.-J. Shyu, "Discrete fractional Hartley and Fourier transforms," IEEE Transactions on Circuits and Systems II: Analog and Digital Signal Processing, vol. 45, no. 6, pp. 665-675, Jun. 1998.

[64] Z. T. Deng, H. J. Caulfield, and M. Schamschula, "Fractional discrete Fourier transforms," Optics Letters, vol. 21, no. 18, pp. 1430-1432, Sep. 1996.

[65] S.-C. Pei and W.-L. Hsue, "The multipleparameter discrete fractional Fourier transform," IEEE Signal Processing Letters, vol. 13, no. 6, pp. 329-332, Jun. 2006

[66] J. G. Vargas-Rubio and B. Santhanam, "On the multiangle centered discrete fractional Fourier transform," IEEE Signal Processing Letters, vol. 12 , no. 4, pp. 273-276, Apr. 2005.

[67] C. Candan, M. A. Kutay, and H. M. Oza- ktas, "The discrete fractional Fourier transform," IEEE Transactions on Signal Processing, vol. 48, no. 5, pp. 1329-1337, May 2000.

[68] C. Candan, "On higher order approximations for Hermite-Gaussian functions and discrete fractional Fourier transforms," IEEE Signal Processing Letters, vol. 14, no. 10, pp. 699-702, Oct. 2007.

[69] S.-C. Pei, W.-L. Hsue, and J.-J. Ding, "Discrete fractional Fourier transform based on new nearly tridiagonal commuting matrices," IEEE Transactions on Signal Processing, vol. 54, no. 10, pp. 3815-3828, Oct. 2006.

[70] M.-H. Yeh and S.-C. Pei, "A method for the discrete fractional Fourier transform computation," IEEE Transactions on Signal Processing, vol. 51, no. 3, pp. 889-891, Mar. 2003.

[71] M. Z. Ikram, K. Abed-Meraim, and Y. Hua, "Fast quadratic phase transform for estimating the parameters of multicomponent chirp signals," Digital Signal Processing, vol. 7, pp. 127$135,1997$.

[72] X.-G. Xia, "Discrete chirp-Fourier transform and its applications to chirp rate estimation," IEEE Transactions on Signal Processing, vol. 48, no. 11, pp. 3122-3233, November 2000.

[73] G. Bi, Y. Wei, G. Li, and C. Wang, "Radix2 DIF fast algorithms for polynomial timefrequency transforms," IEEE Transactions on Aerosp. Electron. Syst., vol. 42, no. 4, pp. 15401546, 2006.

[74] Y. Ju and G. Bi, "Generalized fast algorithms for the polynomial time frequency transforms," IEEE Transactions on Signal Processing, vol. 55, no. 10, pp. 4907-4915, October 2007.

[75] G. Bi, Y. Ju, and X. Li, "Fast algorithms for polynomial time-frequency transforms of realvalued sequences," IEEE Transactions on Signal Processing, vol. 56, no. 5, pp. 1905-1915, May 2008.

[76] M. S. Richman and T. W. Parks, "Understanding discrete rotations," in Proc. of IEEE International Conference on Acoustics, Speech, and Signal Processing (ICASSP-97), vol. 3, Apr. 2124, 1997, pp. 2057-2060.

[77] X. Deng, Y. Li, D. Fan, and Y. Qiu, "A fast algorithm for fractional Fourier transforms," Optics Communications, vol. 138 , no. 4-6, pp. 270-274, Jun. 1997.

[78] H. M. Ozaktas, B. Barshan, and D. Mendlovic, "Convolution and filtering in fractional Fourier domains," Optical Review, vol. 1, no. 1, pp. 1516, Nov. 1994.

[79] M. F. Erden, M. A. Kutay, and H. M. Ozaktas, "Applications of the fractional Fourier transform to filtering, estimation and restoration." in Proc. of the IEEE-EURASIP Workshop on Nonlinear Signal and Image Processing (NSIP'99), Antalya, Turkey, Jun., 20-23 1999, pp. 481-485.

[80] A. Kutay, H. M. Ozaktas, O. Ankan, and L. Onural, "Optimal filtering in fractional Fourier domains," IEEE Transactions on Signal Processing, vol. 45, no. 5, pp. 1129-1143, May 1997.

[81] L. Durak and S. Aldirmaz, "Adaptive fractional Fourier domain filtering," Signal Processing, vol. 90, no. 4, pp. 1188-1196, Apr. 2010. 
[82] I. Djurović, S. Stanković, and I. Pitas, "Digital watermarking in the fractional Fourier transformation domain," Journal of Network and Computer Applications, vol. 24, no. 2, pp. 167-173, Apr. 2001.

[83] F. Q. Yu, Z. K. Zhang, and M. H. Xu, "A digital watermarking algorithm for image based on fractional Fourier transform," in Proc of the 2006 IEEE Conference on Industrial Electronics and Applications, Singapore, May, 24-26 2006, pp. $1-5$.

[84] D. Cui, "Dual digital watermarking algorithm for image based on fractional Fourier transform." in Proc. of the Second Pacific-Asia Conference on Web Mining and Web-based Application (WMWA '09), Wuhan, China, Jun., 6-7 2009 , pp. 51-54.

[85] A. Tefas, N. Nikolaidis, and I. Pitas, Handbook of image and video processing, 2nd ed. Boston, MA, USA: Academic Press, 2005, ch. Watermarking Techniques for Image Authentication and Copyright Protection, pp. 1083-1109.

[86] M. A. Savalonas and S. Chountasis, "Noiseresistant watermarking in the fractional Fourier domain utilizing moment-based image representation," Signal Processing, vol. 90, no. 8, pp. 2521-2528, Aug. 2010

[87] S. Stanković, I. Djurović, and I. Pitas, "Watermarking in the space/spatial-frequency domain using two-dimensional Radon-Wigner distribution," IEEE Transactions on Image Processing, vol. 10 , no. 4, pp. 650-658, Apr. 2001.

[88] D. Simitopoulos, D. E. Koutsonanos, and M. G. Strintzis, "Robust image watermarking based on generalized Radon transformations," IEEE Transactions on Circuits and Systems for Video Technology, vol. 13, no. 8, pp. 732-745, Aug. 2003.

[89] P. H. W. Wong, O. C. Au, and Y. M. Yeung, "Novel blind multiple watermarking technique for images," IEEE Transactions on Circuits and Systems for Video Technology, vol. 13, no. 8, pp. 813-830, Aug. 2003.

[90] G.-R. Feng, L.-G. Jiang, D.-J. Wang, and C. He, "Quickly tracing detection for spread spectrum watermark based on effect estimation of the affine transform," Pattern Recognition, vol. 38, no. 12 , pp. 2530-2536, Dec. 2005.

[91] M. Al-Khassaweneh and S. Aviyente, "Robust watermarking in the Wigner domain," in Proc. of the 2006 IEEE International Conference on Multimedia and Expo, Jul., 9-12 2006, pp. 15571560

[92] T. C. Lu, C. C. Chang, and Y. L. Liu, "A content-based image authentication scheme based on singular value decomposition," Pattern Recognition and Image Analysis, vol. 16, no. 3 , pp. 506-522, Jul. 2006

[93] C.-S. Lu, S.-W. Sun, C.-Y. Hsu, and P.-C Chang, "Media hash-dependent image watermarking resilient against both geometric attacks and estimation attacks based on false positiveoriented detection," IEEE Transactions on Multimedia, vol. 8, no. 4, pp. 668-685, Aug. 2006.

[94] A. Beghdadi and R. Iordache, "Image quality assessment using the joint spatial/spatialfrequency representation," EURASIP Journal on Applied Signal Processing, vol. 2006, pp. 1-8, 2006.

[95] J. Guo, Z. Liu, and S. Liu, "Watermarking based on discrete fractional random transform," Optics Communications, vol. 272, no. 2, pp. 344-348, Apr. 2007.

[96] M. Ozturk, A. Akan, and Y. Cekic, "A robust image watermarking based on time-frequency," in Proc. of the 15th IEEE Signal Processing and Communications Applications Conference (SIU 2007), Eskisehir, Turkey, Jun., 11-13 2007, pp. $1-4$.

[97] A. Bultheel, "Digital watermarking of images in the fractional Fourier domain," Katholieke Universiteit Leuven, Tech. Rep. TW497, Jul. 2007.

[98] H.-B. Sun, G.-S. Liu, H. Gu, and W.-M. Su, "Application of the fractional Fourier transform to moving target detection in airborne SAR," IEEE Transactions on Aerospace and Electronic Systems, vol. 38, no. 4, pp. 1416-1424, Oct. 2002.

[99] A. S. Amein and J. J. Soraghan, "A new chirp scaling algorithm based on the fractional Fourier transform," IEEE Signal Processing Letters, vol. 12 , no. 10 , pp. $705-708$, Oct. 2005.

[100] I. Djurović, T. Thayaparan, and LJ. Stanković, "Adaptive local polynomial Fourier transform in ISAR," EURASIP Journal on Applied Signal Processing, vol. 2006, pp. 1-15, 2006.

[101] M. Martorella, "Novel approach for ISAR image cross-range scaling," IEEE Aerospace and Electronic Systems, vol. 44, no. 1, pp. 281-294, Jan. 2008.

[102] X. Li, G. Bi, and Y. Ju, "Quantitative SNR analysis for ISAR imaging using LPFT," IEEE Transactions on Aerospace and Electronic Systems, vol. 45, no. 3, pp. 1241-1248, Jul. 2009

[103] S. Barbarossa and A. Scaglione, "Autofocusing of SAR images based on the product high-order ambiguity function," IEE Proceedings - Radar, Sonar and Navigation, vol. 145, no. 5, pp. 269273, Oct. 1998

[104] I. Djurović, T. Thayaparan, and LJ. Stanković, "SAR imaging of moving targets using polynomial Fourier transform," IET Signal Processing, vol. 2, no. 3, pp. 237-246, Sep. 2008

[105] V. Popović, I. Djurović, LJ. Stanković, T. Thayaparan, and M. Daković, "Autofocusing of SAR images based on parameters estimated from the PHAF," Signal Processing, vol. 90, no. 5, pp. 1382-1391, May 2010.

[106] I. Djurović, LJ. Stanković, V. Popović, M. Daković, and T. Thayaparan, "Timefrequency analysis for SAR and ISAR imaging," in Proc. of the NATO Advanced Research Workshop on Geographical Information Processing and Visual Analytics for Environmental Security, Trento, Italy, Oct., 13-17 2008, pp. 113127.

[107] LJ. Stanković, "A method for time-frequency analysis," IEEE Transactions on Signal Processing, vol. 42 , no. 1, pp. 225-229, Jan. 1994.

[108] M. Martone, "A multicarrier system based on the fractional Fourier transform for timefrequency-selective channels," IEEE Transactions on Communications, vol. 49, no. 6, pp. 1011-1020, Jun. 2001.

[109] T. Erseghe, N. Laurenti, and V. Cellini, "A 
multicarrier architecture based upon the affine Fourier transform," IEEE Transactions on Communications, vol. 53, no. 5, pp. 853-862, May 2005.

[110] D. Stojanović, I. Djurović, and B. Vojcic, "Interference analysis of multicarrier systems based on affine Fourier transform," IEEE Transactions on Wireless Communications, vol. 8, no. 6, pp. 2877-2880, Jun. 2009.

[111] R. Khanna and R. Saxena, "Improved fractional fourier transform based receiver for spatial multiplexed mimo antenna systems," Wireless Personal Communications, vol. 50, no. 4, pp. 563574, Sep. 2009.

[112] H. K. Lakshminarayana, J. S. Bhat, B. N. Jagadale, and H. M. Mahesh, "Improved chirp modulation spread spectrum receiver based on Fractional fourier transform for multiple access," in Proc. of the 2009 International Conference on Signal Processing Systems, Singapore, May, 1517 2009, pp. 282-286.

[113] I. S. Yetik, M. Kutay, and H. M. Ozaktas, "Image representation and compression with the fractional Fourier transform," Optics Communications, vol. 197, no. 4-6, pp. 275-278, Oct. 2001

[114] C. Vijaya and J. S. Bhat, "Signal compression using discrete fractional Fourier transform and set partitioning in hierarchical tree," Signal Processing, vol. 86, no. 8, pp. 1976-1983, Aug. 2006.

[115] B. Barshan and B. Ayrulu, "Fractional Fourier transform pre-processing for neural networks and its application to object recognition," Neural Networks, vol. 15, no. 1, pp. 131-140, Jan. 2002.

[116] A. W. Lohmann, Z. Zalevsky, and D. Mendlovic, "Synthesis of pattern recognition filters for fractional Fourier processing," Optics Communications, vol. 128, no. 4-6, pp. 199-204, Jul. 1996.

[117] A. Cusmario, "Cryptographic method using modified fractional Fourier transform kernel," US Patent, no. 6718038, April 2004.

[118] S. C. Pei and W.-L. Hsue, "The multipleparameter discrete fractional Fourier transform." IEEE Signal Processing Letters, vol. 13, no. 6, pp. 329-332, June 2006

119] A. M. Youssef, "On the security of a cryptosystem based on multiple-parameters discrete fractional Fourier transform," IEEE Signal Processing Letters, vol. 15, pp. 77-79, 2008.

[120] R. Tao, J. Lang, and Y. Wang, "Optical image encryption based on the multiple-parameter fractional Fourier transform." Optics Letters, vol. 33, no. 6, pp. 581-583, March 2008.

[121] Q. Ran, H. Zhang, J. Zhang, L. Tang, and J. Ma, "Deficiencies of the cryptography based on multiple-parameter fractional Fourier transform," Optics Letters, vol. 34, no. 11, pp. 1729 1731, June 2009.

[122] National Institute of Standards and Technology "Advanced encryption standard (AES)," Federal Information Processing Standards Publication (FIPS 197), Nov. 2001.

[123] S. C. Pei and W.-L. Hsue, "Random discrete fractional Fourier transform," IEEE Sig- nal Processing Letters, vol. 16, no. 12, pp. 10151018, December 2009.

[124] T. Alieva, "Fractional Fourier transform as a tool for investigation of fractal objects," Journal of the Optical Society of America A, vol. 13, no. 6, pp. 1189-1192, Jun. 1996.

[125] Y.-Q. Chen, R. Sun, and A. Zhou, "An improved Hurst parameter estimator based on fractional Fourier transform," in Proc. of the ASME 2007 International Design Engineering Technical Conferences and Computers and Information in Engineering Conference (IDETC/CIE 2007), Las Vegas, Nevada, USA, Sep. 4-7, 2007, pp. $1-11$.

[126] A. C. McBride and F. H. Kerr, "On Namias's fractional Fourier transforms," IMA Journal of Applied Mathematics, vol. 39, no. 2, pp. 159-175, 1987.

[127] G. Dattoli, A. Torre, and G. Mazzacurati, "An alternative point of view to the theory of fractional Fourier transform," IMA Journal of Applied Mathematics, vol. 60, no. 3, pp. 215-224, Jun. 1998.

[128] Y. F. Luchko, H. Matrinez, and J. J. Trujillo, "Fractional Fourier transform and some of its applications," Fractional calculus and applied analysis: An international journal for theory and applications, vol. 11 , no. 4 , pp. 457-470, 2008.

[129] G. Gbur and E. Wolf, "Relation between computed tomography and diffraction tomography," Journal of the Optical Society of America A: Optics, Image Science, and Vision, vol. 18, no. 9, pp. 2132-2137, Sep. 2001.

[130] M. Pineda-Sanchez, M. Riera-Guasp, J. A. Antonino-Daviu, J. Roger-Folch, J. Perez-Cruz, and R. Puche-Panadero, "Diagnosis of induction motor faults in the fractional Fourier domain" IEEE Transactions on Instrumentation and Measurement, vol. 59, no. 8, pp. 2065-2075, Aug. 2010. 